\title{
Large-eddy simulation of cavitating nozzle flow and primary jet break-up
}

\author{
F. Örley, ${ }^{1, a)}$ T. Trummler, ${ }^{1}$ S. Hickel, ${ }^{1,2}$ M. S. Mihatsch, ${ }^{1}$ S. J. Schmidt, ${ }^{1}$ \\ and N. A. Adams ${ }^{1}$ \\ ${ }^{1}$ Institute of Aerodynamics and Fluid Mechanics, Technische Universität München, \\ Boltzmannstr. 15, 85748 Garching bei München, Germany \\ ${ }^{2}$ Chair of Computational Aerodynamics, Faculty of Aerospace Engineering, TU Delft, \\ Kluyverweg 1, 2629 HS Delft, The Netherlands
}

(Received 13 April 2015; accepted 5 August 2015; published online 21 August 2015)

\begin{abstract}
We employ a barotropic two-phase/two-fluid model to study the primary break-up of cavitating liquid jets emanating from a rectangular nozzle, which resembles a high aspect-ratio slot flow. All components (i.e., gas, liquid, and vapor) are represented by a homogeneous mixture approach. The cavitating fluid model is based on a thermodynamic-equilibrium assumption. Compressibility of all phases enables full resolution of collapse-induced pressure wave dynamics. The thermodynamic model is embedded into an implicit large-eddy simulation (LES) environment. The considered configuration follows the general setup of a reference experiment and is a generic reproduction of a scaled-up fuel injector or control valve as found in an automotive engine. Due to the experimental conditions, it operates, however, at significantly lower pressures. LES results are compared to the experimental reference for validation. Three different operating points are studied, which differ in terms of the development of cavitation regions and the jet break-up characteristics. Observed differences between experimental and numerical data in some of the investigated cases can be caused by uncertainties in meeting nominal parameters by the experiment. The investigation reveals that three main mechanisms promote primary jet break-up: collapse-induced turbulent fluctuations near the outlet, entrainment of free gas into the nozzle, and collapse events inside the jet near the liquid-gas interface. (C) 2015 AIP Publishing LLC. [http://dx.doi.org/10.1063/1.4928701]
\end{abstract}

\section{INTRODUCTION}

Future emission standards for automotive engines demand for a more efficient mixing and combustion process. Modern fuel injection systems play a key role in the optimization of the mixing process of fuel with air in the combustion chamber. Recent developments aim at increasing the injection rail pressures, which enhances jet break-up and mixing and hence improves the combustion. Higher flow accelerations, however, imply thermo-hydrodynamic effects, such as cavitation which occurs when the pressure drops locally below saturation conditions and the liquid vaporizes. ${ }^{1}$ The subsequent collapse of such vapor structures when convected into regions of higher pressure causes the emission of strong shock-waves. Near walls also high-velocity liquid jets directed towards the wall surface are created. ${ }^{2-5}$ Structure loads induced by such phenomena lead to material erosion, but are also employed to clean injection nozzles from surface deposits, ${ }^{6}$ and can promote primary jet break-up. ${ }^{7-10}$ Furthermore, flow cavitation can lead to choked conditions in a nozzle and thus maintains a pressure-drop independent mass flow rate. ${ }^{11}$ Surface erosion due to cavitation may be so strong that injection performance degrades severely or devices may fail. ${ }^{12,13}$

A detailed understanding of flow phenomena is necessary to control the effects of cavitation and their influence on jet and spray characteristics when injected into a gas phase. Typically,

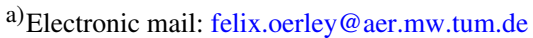


characteristic dimensions of fuel nozzles inside diesel injectors are in the range of tens to several hundred micrometers. This makes the instrumentation of an injection nozzle with diagnostic equipment, such as optical measurement methods or pressure sensors, for an experimental flow characterization, a challenging task. ${ }^{14,15}$ Another limiting factor are high operating pressures. Short intrinsic time scales imposed by inherent flow dynamics, by functional components such as opening or closing of control valves or the injector needle, or by multiple injections per engine cycle make time-accurate measurements challenging. Therefore, experimental analyses assessing cavitation erosion can supply information about incubation time, position, and the progress of erosion damage,${ }^{16}$ but do not necessarily reveal all aspects of the underlying flow dynamics, which would be desirable for the identification of possible countermeasures and optimization. Computational Fluid Dynamics (CFD), on the other hand, can provide time-resolved information on flow structures in arbitrary small geometries. Numerical simulations of cavitating flows thus have become an important tool in the design process.

Based on the pioneering work of Plesset et al. ${ }^{17,18}$ on the growth and collapse of isolated bubbles for different boundary conditions, d'Agostino and Brennen ${ }^{19}$ and Delale et al. ${ }^{20}$ developed 1-D models of bubbly liquids. Since then, various cavitation models have been proposed, which can be categorized into two main groups: two-fluid models and single-fluid models. With two-fluid models, an individual set of conservation equations is solved for each species. Eulerian-Eulerian two-fluid models incorporate a mass transfer due to phase transition between liquid and vapor phases at the phase boundary, which can be tracked by a sharp-interface method as proposed by, e.g., Lauer et al. ${ }^{21,22}$ Eulerian-Lagrangian two-fluid cavitation models, as investigated by Giannadakis et al., ${ }^{23,24}$ consider the liquid as a continuous carrier phase in a Eulerian frame of reference, whereas single vapor bubbles or parcels of bubbles are tracked as Lagrangian elements using the Rayleigh-Plesset equation for growth and collapse. In contrast, single-fluid cavitation models, which are sometimes referred to as homogeneous Eulerian models, treat the fluid as a continuous mixture of liquid and vapor solving a single set of constitutive equations characterized by average mixture properties, such as mixture density and mixture viscosity. Although not necessary, the void fraction can be transported separately as with Volume-of-Fluid (VOF) methods, ${ }^{25}$ allowing for inclusion of non-equilibrium phase transition effects as proposed by Kunz et al. ${ }^{26}$ or Yuan et al. ${ }^{27}$ Assuming the liquid and gaseous phases to be in thermal and mechanical equilibrium, Schmidt et al. ${ }^{28,29}$ and Schnerr et al $^{30}$ developed a model in which the two phases are uniformly distributed within each cell without slip between the liquid and vapor phases, neglecting surface tension and buoyancy effects. Thermodynamic equilibrium models exhibit an intrinsic length-scaling capability ${ }^{31}$ and thus are particularly suitable for application to complex flows in conjunction with subgrid-scale (SGS) models for non-resolved flow scales. ${ }^{32}$

Early studies of cavitating flows of submerged injection nozzles ${ }^{33,34}$ were performed by means of solving the incompressible Reynolds-Averaged Navier-Stokes (RANS) equations. Duke et al. ${ }^{35}$ expect that large vortical structures within the nozzle flows strongly interact with cavitation structures and therefore suggest to perform Large-Eddy Simulations (LESs). Recent studies of Salvador et $a l .{ }^{36}$ and Sou et $a l .{ }^{37}$ present incompressible LES of turbulent, cavitating flows using a Euler-Lagrangian Rayleigh-Plesset model. Schnerr et al. ${ }^{30}$ present a compressible framework for the simulation of cavitating flows. This method resolves wave dynamics and thus can predict also acoustic cavitation and captures instantaneous pressure fields.

Very challenging in the context of injection of liquid jets into a combustion chamber is the mutual interaction of cavitating liquids and non-condensable gas. Sou et al. ${ }^{38,39}$ and Som and Aggarwal $^{40}$ assume that collapse events of cavitation structures near the nozzle outlet may enhance turbulent fluctuations, which in turn promote primary break-up of the liquid jet. This effect is observed at a supercavitating state of the nozzle, i.e., when stable cavitation sheets reach from the inlet edge of the nozzle to the outlet region, see also Shibata et al. ${ }^{41}$ A simultaneous simulation of the cavitating nozzle flow and the liquid jet is necessary to capture the effect of cavitation structures on jet break-up.

Recently, simulations of breaking liquid jets were presented by Menard et al ${ }^{42}$ and Desjardins and Pitsch $^{43}$ in an Eulerian framework with a VOF method. The authors apply the method only to external flows. To assess the impact of two-phase nozzle flow, additional simulations of nozzle 
flows are required. Lagrangian particle methods introduced by Dukowicz ${ }^{44}$ significantly reduce the required mesh resolution and are adopted by Som et al. ${ }^{45}$ who consequently consider the effects from nozzle flows when coupling with a subsequent spray simulation. To correct for potential inconsistencies of the two-phase models in the two separate simulations, a combination of the models denoted as Eulerian-Lagrangian-Spray and Atomization (ELSA) model by Blokkeel et al. ${ }^{46}$ switches from a Eulerian description of the nozzle flow to a Lagrangian particle simulation of liquid droplets in the break-up region. This approach, however, does not incorporate compressibility effects inside the liquid. Wang et al. ${ }^{47}$ propose an eight-equation model based on the work of Saurel et al., ${ }^{48,49}$ to assess both internal and external 2-D nozzle flows simultaneously including cavitation effects by a stiffened gas equation of state, whereas gas and vapor are represented as one single miscible phase. In their recent work, Battistoni et al.$^{50}$ compare a non-equilibrium thermal mixture model with a Eulerian multi-fluid description with Rayleigh bubble dynamics for phase change. In their simulations of cavitating nozzle flows, a free gas content was considered, but effects on the primary jet break-up were not assessed. Ishimoto et al..$^{51}$ performed the first fully coupled simulation of cavitating nozzle flow and jet break-up with a fully compressible, barotropic LES model based on a VOF method.

In this work, we propose a simple, closed-form barotropic two-fluid cavitation model including non-condensable gases. The thermodynamic model is an extension of the compressible framework for single-fluid implicit large-eddy simulations of turbulent, cavitating flows, recently presented by Egerer et al. ${ }^{32}$ Compared to many previous approaches, such a monolithic, Eulerian description of cavitating liquid and gas enables the analysis of nozzle and jet flows in one single simulation without domain-coupling issues or parameter calibration. Wave dynamic effects, which may propagate from the nozzle into the jet, and vice-versa, are fully represented. The framework allows to easily integrate fluid models with different properties and complexity for a broad range of applications. The model is used in this paper to simulate an experimental reference configuration of Sou et al. ${ }^{38,39}$ for a cavitating water jet injected into ambient air.

The paper is structured as follows. First, the governing equations and thermodynamic models are introduced in Sec. II. The numerical method applied for turbulence modelling is briefly discussed in Sec. III. In Sec. IV, we present the experimental and computational setup. Finally, in Sec. V, we show results for a turbulent, cavitating nozzle slot flow injected into ambient air and identify main mechanisms for primary jet break-up. Conclusions are drawn in Sec. VI.

\section{MATHEMATICAL AND PHYSICAL MODEL}

\section{A. Governing equations}

We consider the three-dimensional, fully compressible Navier-Stokes equations in conservation form

$$
\frac{\partial \mathbf{U}}{\partial t}+\nabla \cdot \mathbf{F}(\mathbf{U})=0
$$

The state vector $\mathbf{U}=\left[\rho, \rho u_{1}, \rho u_{2}, \rho u_{3}, \rho \xi_{G}\right]$ contains the conserved variables mixture density $\rho$, momentum $\rho \mathbf{u}$, and gas density $\rho \xi_{G}$. The transport equation for the total energy is not needed, because the thermodynamic model follows a barotropic equation of state, see Sec. II B. Accordingly, heat transfer is neglected. However, latent heat of evaporation is taken into account in the barotropic equation of state. The numerical flux vector $\mathbf{F}(\mathbf{U})$ is split into convective $\mathbf{C}(\mathbf{U})$ and viscous $\mathbf{S}(\mathbf{U})$ parts as

$$
\mathbf{F}(\mathbf{U})=\mathbf{C}(\mathbf{U})+\mathbf{S}(\mathbf{U})
$$

with

$$
\mathrm{C}_{i}(\mathbf{U})=u_{i} \mathbf{U}
$$

and

$$
\mathrm{S}_{i}(\mathbf{U})=\left[0, \delta_{i 1} p-\tau_{i 1}, \delta_{i 2} p-\tau_{i 2}, \delta_{i 3} p-\tau_{i 3}, 0\right]
$$


Here, $p$ is the static pressure, $\delta_{i j}$ is the Kronecker delta, and $\tau_{i j}$ denotes the viscous stress tensor

$$
\tau_{i j}=\mu_{m i x}\left(\partial_{j} u_{i}+\partial_{i} u_{j}-\frac{2}{3} \delta_{i j} \partial_{k} u_{k}\right),
$$

with $\mu_{m i x}$ being the dynamic mixture viscosity of the fluid.

\section{B. Thermodynamic model}

The homogeneous-mixture single-fluid model for cavitation is extended by a component of non-condensable gas to a two-fluid mixture model. The single-fluid cavitation model has been extensively validated in previous studies, e.g., for collapsing bubble clouds by Schmidt et al. ${ }^{31}$ LES of cavitating, turbulent, wall-bounded water, and fuel flows by Hickel et al. ${ }^{52}$ and Egerer et al. ${ }^{32}$ and closing control valves by Örley et al. ${ }^{53}$ In the following, the thermodynamic models for both cavitating liquid and gas are introduced, followed by a description of the coupled methodology.

In Fig. 1, the principle of the homogeneous-mixture model inside a computational cell in the framework of a finite volume discretization is sketched. The actual interface of water in its liquid and vaporous state, which may consist of several discrete small vapor bubbles inside one computational cell, and the interface between liquid (or liquid-vapor) and non-condensable gas are not reconstructed, unlike with sharp-interface methods, e.g., Lauer et al. ${ }^{21}$ Surface tension is thus neglected. Instead, we consider the cell-averaged solution assuming negligible slip between the phases, i.e., $u=u_{L}=u_{M}=u_{G}$, and equal pressure $p=p_{L}=p_{M}=p_{G}$. The pure liquid, liquid-vapor, and non-condensable gas components are denoted with subscript $L, M$, and $G$, respectively. The volume fraction of component $\Phi=\{L, M, G\}$ inside a control volume $V$ is

$$
\beta_{\Phi}=\frac{V_{\Phi}}{V}, \text { with } \sum_{\Phi} \beta_{\Phi}=1 .
$$

Accordingly, the mass fraction with respect to mass $m$ is defined as

$$
\xi_{\Phi}=\frac{m_{\Phi}}{m}, \text { with } \sum_{\Phi} \xi_{\Phi}=1 .
$$

The density $\rho_{\Phi}$ of component $\Phi$ can be written as

$$
\rho_{\Phi}=\frac{m_{\Phi}}{V_{\Phi}}=\frac{\xi_{\Phi} m}{\beta_{\Phi} V}=\frac{\xi_{\Phi}}{\beta_{\Phi}} \rho,
$$

and the averaged mixture density is

$$
\rho=\frac{m}{V}=\sum_{\Phi} \beta_{\Phi} \rho_{\Phi} .
$$

By using barotropic thermodynamic closure relations for each phase, the equation of state can be formulated in a suitable way to solve for the cell-averaged pressure $p=p(\rho)$. Hence, mixtures of

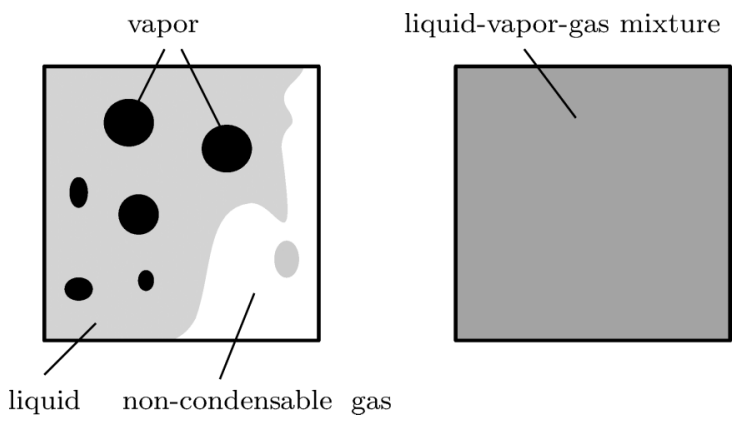

FIG. 1. Homogeneous-mixture model inside a computational cell of a finite volume discretization: resolved phase interfaces (left); numerical approximation (right). 
non-condensable gas and pure vapor/pure liquid may occur locally, since we differentiate between purely liquid and vapor phases in water only based on the local pressure. Inter-diffusion of fluids occurs only due to turbulent mixing and numerical diffusion. sound

Liquid water and water vapor are modelled based on the definition of the isentropic speed of

$$
c^{2}=\left.\frac{\partial p}{\partial \rho}\right|_{s=\text { const }} .
$$

Assuming a constant speed of sound $c=$ const for each phase, integration of Eq. (10) from saturation density to the cell averaged density leads to a linearized equation of state

$$
\rho=\rho_{s, l i q}+\frac{1}{c^{2}}\left(p-p_{s}\right),
$$

where subscript $s$ denotes thermodynamic quantities at saturation conditions. We use constant values for saturation pressure $p_{s}=2340 \mathrm{~Pa}$ and saturation density of the liquid $\rho_{s, l i q}=998.1618 \mathrm{~kg} / \mathrm{m}^{3}$ at a reference temperature $T_{\text {ref }}=293.15 \mathrm{~K}$.

For purely liquid water, i.e., $p>p_{s}$, the speed of sound is $c=c_{L}=1482.35 \mathrm{~m} / \mathrm{s}$ at ambient conditions. Comparison to more accurate models, such as the modified Tait equation proposed by Saurel et al.,${ }^{54}$ or NIST data for liquid water, ${ }^{55}$ shows negligible deviation even for high pressures, as shown in Fig. 2. The error of the linearized equation of state relative to accurate NIST data for liquid water ${ }^{55}$

$$
\varepsilon=\frac{\rho_{\text {NIST }}-\rho_{\text {lin }}}{\rho_{\text {NIST }}}
$$

is shown in Table I for a pressure range up to 2000 bar. In our study liquid pressures reach approximately 3.5 bars at the inlet, while collapse pressures for strong events exceed 1500 bar.

Assuming a high concentration of nuclei, a liquid starts to evaporate where the local pressure drops below a critical value, i.e., $p<p_{c}$. A straightforward approach is to use the saturation pressure of the liquid phase ${ }^{27,34}$ as the critical pressure, i.e.,

$$
p<p_{s} .
$$

We assume that phase change occurs instantaneously and in local thermodynamic equilibrium. In this case, the same equation of state as introduced in Eq. (11) may be used, but a different speed of sound is employed. Franc et al. ${ }^{56}$ derive the equilibrium speed of sound in the two-phase region as

$$
\frac{1}{\rho c^{2}}=\frac{\alpha}{\rho_{v} c_{v}^{2}}+\frac{1-\alpha}{\rho_{l} c_{l}^{2}}-\frac{(1-\alpha) \rho_{l} c_{p, l} T}{\left(\rho_{v} L\right)^{2}} .
$$

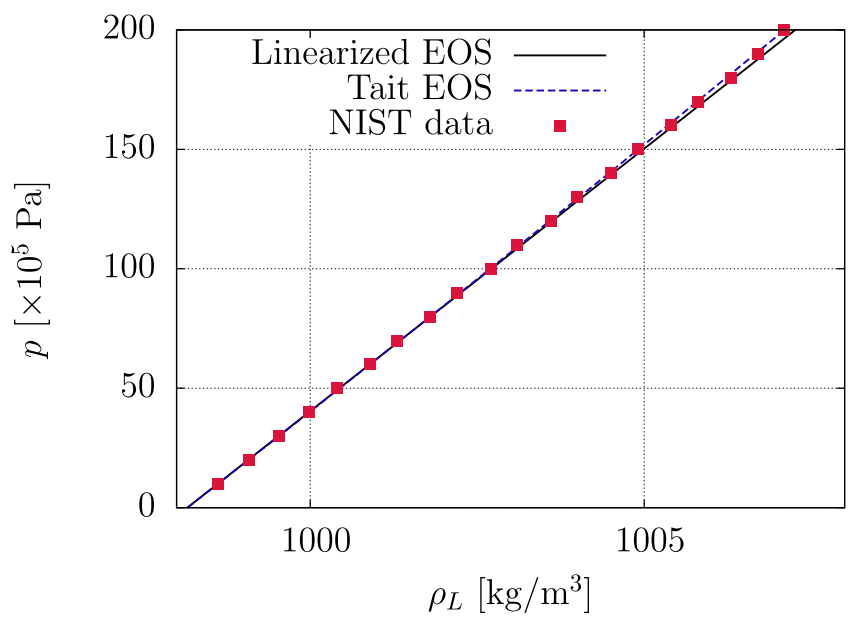

FIG. 2. Comparison of linearized equation of state for liquid water to modified Tait equation ${ }^{54}$ and NIST data. ${ }^{55}$ 
TABLE I. Relative error of linearized equation of state.

\begin{tabular}{lrrr}
\hline \hline $\begin{array}{l}\text { Pressure } p \\
\left(10^{5} \mathrm{~Pa}\right)\end{array}$ & $\begin{array}{r}\rho_{N I S T}(p)^{55} \\
\left(\mathrm{~kg} / \mathrm{m}^{3}\right)\end{array}$ & $\begin{array}{r}\rho_{\text {lin }}(p) \\
\left(\mathrm{kg} / \mathrm{m}^{3}\right)\end{array}$ & $|\varepsilon|(\%)$ \\
\hline 1.0 & 998.207 & 998.206 & 0.00038 \\
10.0 & 998.620 & 998.616 & 0.00042 \\
100.0 & 1002.692 & 1002.712 & 0.00116 \\
2000.0 & 1071.433 & 1089.179 & 1.41331 \\
\hline
\end{tabular}

Neglecting the last term results in the "frozen" speed of sound. Franc et al. provide numerical values for the two different definitions of the speed of sound at a void fraction of $50 \%$ in water, for the equilibrium state $c_{e q}=0.08 \mathrm{~m} / \mathrm{s}$, and for the frozen state $c_{\text {frozen }}=3 \mathrm{~m} / \mathrm{s}$. Brennen ${ }^{1}$ finds that including phase-change effects results in a speed of sound which lies between these two bounds.

We consider an average of the speed of sound between a frozen and an equilibrium isentropic phase change in the two-phase region. We use a numerical value of $c=c_{M}=1 \mathrm{~m} / \mathrm{s}$ in the two-phase region, which satisfies the observation of Brennen.

The combined equation of state for the liquid and liquid-vapor fluid components, which is denoted with $L M$, can be summarized as

$$
\rho_{L M}=\rho_{s, l i q}+\frac{1}{c^{2}}\left(p-p_{s}\right), c=\left\{\begin{array}{ll}
c_{L}, & p \geq p_{s} \\
c_{M}, & p<p_{s}
\end{array} .\right.
$$

The non-condensable gas phase is modelled as an isothermal, ideal gas

$$
\rho_{G}=\frac{p}{\mathrm{R}_{G} T_{r e f}}
$$

at reference temperature $T_{r e f}$. $\mathrm{R}_{G}$ denotes the specific gas constant, we use $\mathrm{R}_{G}=287.06 \mathrm{~J} /(\mathrm{kg} \mathrm{K})$ for air. The speed of sound for air at reference temperature is

$$
c_{G}=\sqrt{\kappa \mathrm{R}_{G} T_{\text {ref }}}=343.24 \mathrm{~m} / \mathrm{s},
$$

with the ratio of specific heats $\kappa=1.4$.

To obtain a closed form equation of state for all three phases, the individual closure relations for each phase are combined via Eq. (9) to

$$
\rho=\beta_{L M}\left[\rho_{s}+\frac{1}{c^{2}}\left(p-p_{s}\right)\right]+\beta_{G} \frac{p}{\mathrm{R}_{G} T_{r e f}}, c=\left\{\begin{array}{ll}
c_{L}, & p \geq p_{s} \\
c_{M}, & p<p_{s}
\end{array} .\right.
$$

Following Eq. (6), which leads to the relation $\beta_{L M}=1-\beta_{G}$ for a two-fluid mixture, Eq. (18) can be written as a function of the gas mass fraction $\xi_{G}$ using Eq. (8) as

$$
\rho=\left(1-\xi_{G} \frac{\rho \mathrm{R}_{G} T_{r e f}}{p}\right)\left(\rho_{s, l i q}+\frac{1}{c^{2}}\left(p-p_{s}\right)\right)+\xi_{G} \rho .
$$

The mixture pressure $p$ is now obtained by solving the quadratic equation. The volume fraction of the liquid/liquid-vapor phase $\beta_{L M}$ is computed from Eq. (8). In practice, we first solve for $p$ for water in a purely liquid state, i.e., the speed of sound is set to $c=c_{L}$. If no solution is found, we repeat this step for $c=c_{M}$.

Finally, the local vapor volume fraction can be computed from

$$
\alpha_{v}=\frac{V_{\text {vap }}}{V}=\left\{\begin{array}{ll}
0, & \rho \geq \rho_{s, l i q} \\
\beta_{L M} \frac{\rho_{s, l i q}-\rho_{L M}}{\rho_{s, l i q}-\rho_{s, v a p}}, & \rho<\rho_{s, l i q}
\end{array},\right.
$$

$V_{\text {vap }}$ denotes the part of the volume $V$ that is occupied by vapor and $\rho_{s, v a p}$ is the saturation density pure vapor, respectively. 
Viscosity in the liquid is considered following Bensow and Bark. ${ }^{57}$ The mixture viscosity is defined as

$$
\mu_{\text {mix }}=\beta_{L M}\left[\left(1-\alpha_{v}\right) \mu_{\text {liq }}+\alpha_{v} \mu_{\text {vap }}\right]+\beta_{G} \mu_{G},
$$

where the viscosities for the liquid, vapor, and gas phases at the reference temperature are $\mu_{\text {liq }}=$ $1.002 \times 10^{-3} \mathrm{~Pa} \mathrm{~s}, \mu_{\text {vap }}=9.727 \times 10^{-6} \mathrm{~Pa} \mathrm{~s}$, and $\mu_{G}=18.24 \times 10^{-6} \mathrm{~Pa} \mathrm{~s}$, respectively.

\section{NUMERICAL METHOD}

In turbulent flows, the smallest scales account for a large part of the energy dissipation and therefore play a key role in the macroscopic flow behaviour. Since with LES the smallest scales are not resolved on the computational grid, effects of these unresolved SGSs must be modelled. We employ an implicit LES approach based on the Adaptive Local Deconvolution (ALDM) method by Adams et al. ${ }^{58}$ and Hickel et al..$^{59,60}$ In contrast to explicit SGS models, which are often used for LES of cavitating flows, implicit LES merges turbulence modelling and numerical discretization of the conservation equations. ALDM is a nonlinear finite volume method and incorporates free parameters that control the implicit SGS model. A SGS model that is consistent with turbulence theory is obtained through parameter calibration, see Refs. 59, 61, and 62 for details. The compressible version of ALDM proposed by Hickel et al. ${ }^{60,77}$ can capture shock waves while smooth pressure waves and turbulence are propagated without excessive numerical dissipation. More details on the validation of ALDM for cavitating flows are given in Egerer et al. ${ }^{32}$ In this paper, the ALDM reconstruction is applied only to the momentum equation. Density and free-gas mass fraction are discretized by a second order upwind biased reconstruction with van Albada limiter. ${ }^{63}$ The viscous flux is discretized by a linear second-order centered scheme. Time integration is performed with an explicit, third-order Runge-Kutta method of Gottlieb and Shu. ${ }^{64}$

\section{LES OF CAVITATING NOZZLE JETS}

\section{A. Experimental setup}

The setup presented by Sou et al. ${ }^{38,39}$ can be regarded as a large-scale, generic fuel injector as found in many automotive applications. A schematic of the setup is shown in Fig. 3. Dimensions are summarized in Table II. The rectangular nozzle geometry consists of acrylic flat plates as front and back walls to gain optical access and thin stainless steel plates of width $W_{N}=1 \times 10^{-3} \mathrm{~m}$ to form the nozzle geometry of height $H_{N}=4 \times 10^{-3} \mathrm{~m}$ and length $L_{N}=16 \times 10^{-3} \mathrm{~m}$. Upstream of the nozzle section, the duct height is $H_{C}=32 \times 10^{-3} \mathrm{~m}$, which corresponds to a contraction ratio of 8:1 and can thus be regarded as a slot flow. The duct opens into a large reservoir of quiescent ambient air. In the following, we refer to the symmetry line crossing the nozzle inlet plane as the origin of an underlying Cartesian coordinate system.

Tap water at $T_{w}=292 \mathrm{~K}$ was processed through an air separation tank and used as working fluid. A plunger pump was used to control the flow rate and to prescribe a cavitation number

$$
\sigma=\frac{p_{\infty}-p_{s}}{0.5 \rho_{l i q, \infty} U_{N}^{2}}
$$
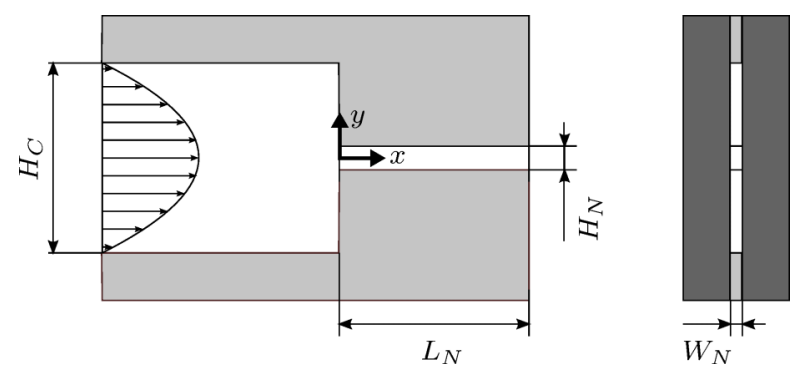

FIG. 3. Schematic of the nozzle: side view (left), back view (right). 
TABLE II. Geometric dimensions of the nozzle geometry.

\begin{tabular}{lcccc}
\hline \hline Parameter & $H_{N}$ & $L_{N}$ & $W_{N}$ & $H_{C}$ \\
\hline Value $\left(10^{-3} \mathrm{~m}\right)$ & 4.0 & 16.0 & 1.0 & 32.0 \\
\hline \hline
\end{tabular}

where $p_{\infty}=1 \mathrm{~atm}=1.01325 \times 10^{5} \mathrm{~Pa}$ corresponds to the surrounding pressure, $\rho_{\text {liq, } \infty}$ is the liquid density at ambient conditions, and $U_{N}$ is the mean streamwise liquid velocity inside the nozzle. This non-dimensional parameter relates the pressure difference of the surrounding and vapor pressure to the local dynamic pressure. The Reynolds number is defined as

$$
\operatorname{Re}=\frac{U_{N} H_{N}}{v_{L}},
$$

with the liquid kinematic viscosity $v_{L}$. Furthermore, we define the non-dimensional cavitation length $L_{c}^{*}$ as the ratio of the mean streamwise extent of the cavitation zone and the nozzle length $L_{N}$. The Weber number is

$$
W e=\frac{\rho_{\text {liq, }, \infty} U_{N}^{2} H_{N}}{\sigma_{s}},
$$

where $\sigma_{s}$ is the surface tension coefficient.

Sou et $a l . .^{38,39}$ investigated a variety of cavitation numbers $\sigma=\{0.65,0.78,1.27\}$. The nondimensional parameters for the operation points investigated in this numerical study are provided in Table III. The non-condensable gas-to-liquid dynamic viscosity ratio is $\eta=\mu_{g a s, \infty} / \mu_{l i q, \infty}=$ $1.82 \times 10^{-2}$ and the non-condensable gas-to-liquid density ratio is $\lambda=\rho_{\text {gas }, \infty} / \rho_{\text {liq, } \infty}=1.21 \times 10^{-3}$. Note that, since surface tension is not included in our present model and the Weber number thus becomes infinity, we only focus on primary break-up, which is driven by inertia. This simplification is supported by the low non-condensable gas-to-liquid density and viscosity ratio, and the high Reynolds numbers. We do not consider secondary break-up and atomization, which is significantly affected by surface tension, in this work.

In Table IV, the cavitation and jet characteristics observed in the experiment for the different cases selected for this study are summarized. For cavitation numbers $\sigma>1.2$, no cavitation was observed $\left(L_{c}^{*}=0\right)$ and the jet was characterized as "wavy jet." For $0.75 \leq \sigma \leq 1.2$, cavitation zones at the inlet region of the nozzle started to develop $\left(L_{c}^{*} \sim 0.2-0.4\right)$, while the liquid jet characteristics were not altered. A further decrease in the cavitation number to $0.55<\sigma<0.75$ led to a growth of the cavitation zones almost up to the whole nozzle length $\left(L_{c}^{*} \sim 0.8-0.9\right)$, and enhanced primary jet break-up and jet atomization was observed.

Optical data were sampled by transmitted light imaging. Furthermore, quantitative data for mean velocity and velocity fluctuations are available from Laser Doppler Velocimetry (LDV) measurements. The water was seeded with silicone carbide particles. The effect of the particles on the flowfield was found to be negligible. ${ }^{38}$

\section{B. Computational setup}

The computational domain used in our study is sketched in Fig. 4 showing the block structure. The duct is connected to a large outlet region of $W_{O}=160 \times W_{N}, H_{O}=75 \times H_{N}$, and

TABLE III. Experimental conditions investigated by Sou et al. ${ }^{38,39}$

\begin{tabular}{lccc}
\hline \hline $\begin{array}{l}\text { Mean liquid } \\
\text { velocity } U_{N}(\mathrm{~m} / \mathrm{s})\end{array}$ & $\begin{array}{c}\text { Cavitation } \\
\text { number } \sigma(-)\end{array}$ & $\begin{array}{c}\text { Reynolds } \\
\text { number } \operatorname{Re}(-)\end{array}$ & $\begin{array}{c}\text { Weber } \\
\text { number We (-) }\end{array}$ \\
\hline 12.5 & 1.27 & 50000 & 8562 \\
16.0 & 0.78 & 64000 & 14029 \\
17.5 & 0.65 & 70000 & 16783 \\
\hline \hline
\end{tabular}


TABLE IV. Cavitation and jet characteristics. ${ }^{38,39}$

\begin{tabular}{lll}
\hline \hline $\begin{array}{l}\text { Cavitation } \\
\text { number } \sigma(-)\end{array}$ & \multicolumn{1}{c}{$\begin{array}{c}\text { Cavitation } \\
\text { characteristics }\end{array}$} & $\begin{array}{c}\text { Jet } \\
\text { characteristics }\end{array}$ \\
\hline 1.27 & No cavitation & Wavy jet \\
0.78 & Developing cavitation & Wavy jet \\
0.65 & Supercavitation & Spray \\
\hline \hline
\end{tabular}

$L_{O}=17.5 \times L_{N}$, which is employed to resemble the injection into free ambient air while avoiding any influence of the boundary conditions.

A total of $\Delta x=24 \times 10^{-3} \mathrm{~m}$ of the inlet duct are computed to avoid spurious influence of the inlet boundary condition. At the inlet, we prescribe a purely liquid water flow, i.e., $\xi_{G \text {,in }}=0.0$. If not stated otherwise, we set a laminar, doubly parabolic stream-wise velocity profile

$$
u=\frac{9}{4} U_{B}\left[1-\left(\frac{y}{H_{C} / 2}\right)^{2}\right]\left[1-\left(\frac{z}{W_{N} / 2}\right)^{2}\right] .
$$

The bulk velocity, $U_{B}=U_{N} / 8$ according to the ratio of slot and inlet area, is set to match the corresponding Reynolds number for each operating point. We use Neumann conditions for the pressure at the inflow boundary and the density is computed from Eq. (15). At the outlet, we apply a static pressure boundary condition $p(y, z)=\left(p_{\text {out }}+p_{\mathrm{lc}}(y, z)\right) / 2$, which is computed from the static pressure in the last cell layer, $p_{\mathrm{lc}}(y, z)$, and the prescribed pressure $p_{\text {out }}=1 \mathrm{~atm}$. This methodology reduces spurious reflections at the outlet while asymptotically maintaining the desired outlet pressure. All other quantities are extrapolated linearly. Walls are treated as adiabatic with no-slip condition for the velocity components and Neumann conditions for all other quantities. The domain is initialized at rest and is filled with purely liquid water without non-condensable gas component (i.e., $\xi_{G}=0$ ) inside the nozzle for $x<16 \times 10^{-3} \mathrm{~m}$ and gas (i.e., $\xi_{G}=1$ ) for $x \geq 16 \times 10^{-3} \mathrm{~m}$ in the outlet region. The initial pressure is set to $p_{\text {init }}=1 \mathrm{~atm}$.

We discretize the domain on a Cartesian block-structured mesh. To reduce computational cost, we employ a successive grid refinement in the nozzle and near nozzle inlet and outlet during the simulation. For each case, initially we let the mean flow develop over a long period of time of approximately 100 flow-through times of the nozzle on a coarse grid, which consists of roughly $2.7 \times 10^{6}$ cells. As soon as the flow has developed, we refine the grid over several intermediate levels until the final grid resolution is reached. We ensure a steady or periodic signal of the global

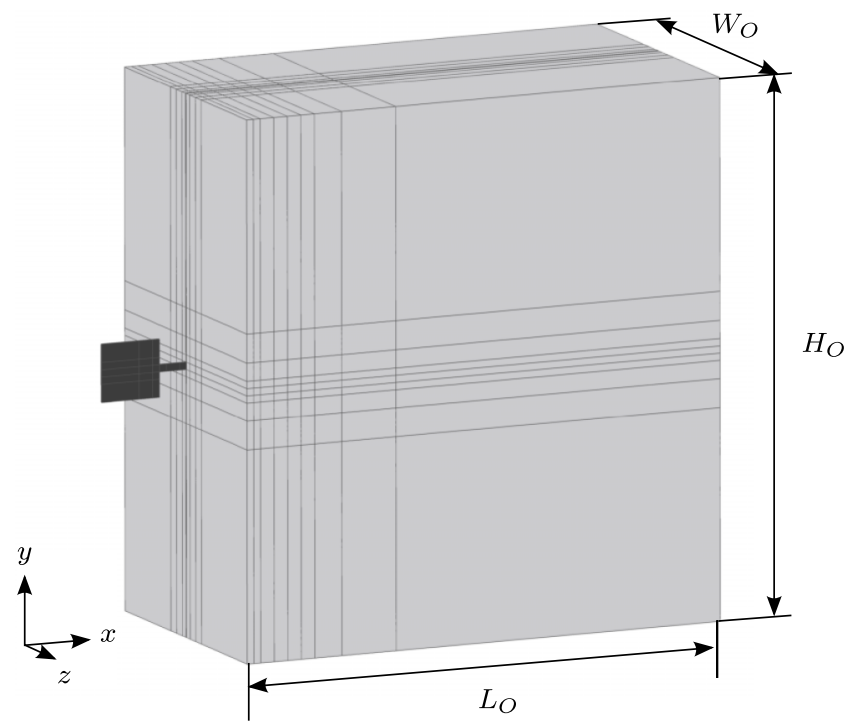

FIG. 4. Computational domain and block structure of the quasi two-dimensional throttle (shaded dark gray) connected to a large outflow reservoir (shaded light gray). 

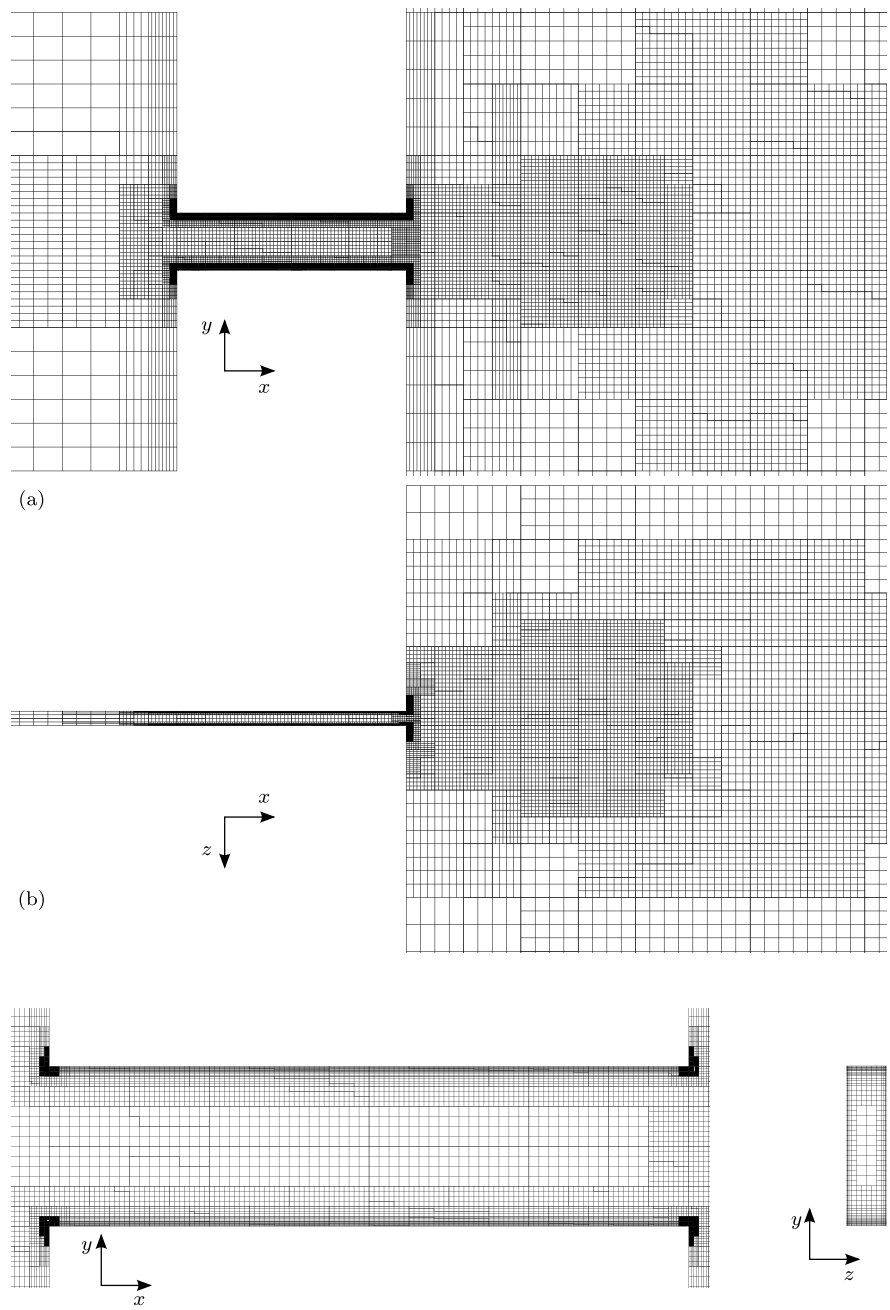

(c)

(d)

FIG. 5. Computational grid of the full computational domain: (a) $x-y$ view in the $z$ symmetry plane; (b) $x-z$ view in the $y$ symmetry plane; (c) $x-y$ view in the $z$ symmetry plane of the nozzle region; (d) $y$ - $z$ view in the nozzle cross section. Only every fourth grid line is shown.

vapor mass fraction at each level before refining the grid. In every step, the grid is refined near the nozzle wall and outflow region by a ratio of 2:1, and the solution of the previous level is interpolated onto the refined grid. At grid interfaces of different resolution, we apply a conservative interpolation procedure. We propagate the flow solution on three intermediate grids before sampling data on the fine grid. The fine grid, which is shown in Fig. 5, contains $43.1 \times 10^{6}$ cells with a smallest cell size of $3.91 \times 10^{-6} \mathrm{~m}$ at the nozzle walls and at the inlet and outlet edges. An analysis on the grid convergence behavior is given in Appendix A.

Three-dimensional statistical data of the flow field were sampled every 100 computational time steps $\left(\Delta t_{s} \approx 0.6 \times 10^{-7} \mathrm{~s}\right)$, which corresponds to a sampling frequency of $f_{s} \approx 14 \mathrm{MHz}$, over an interval of at least $\Delta t_{\text {avg }}=3 \times 10^{-3} \mathrm{~s}$, which corresponds to approximately three flow-through times of the nozzle. Time averaged quantities are denoted by $\langle\bullet\rangle$.

\section{RESULTS AND DISCUSSION}

\section{A. Comparison of nozzle flow with experimental data}

We first compare our numerical results with the available experimental data for three operating points. Figure 6 shows transmitted light images of instantaneous vapor structures observed in the 


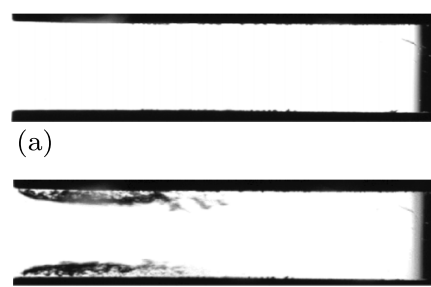

(d)

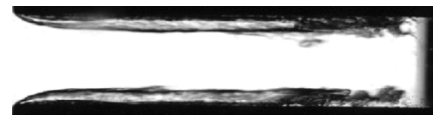

(g)

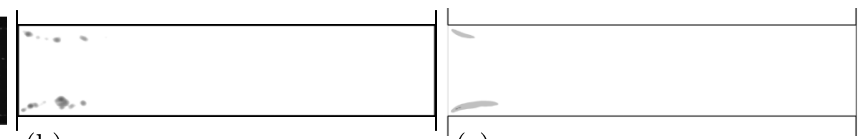

(b)

(c)

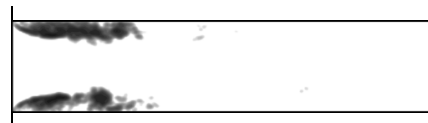

(e)

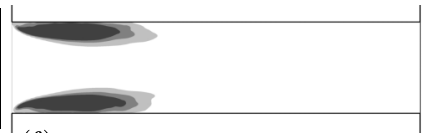

(f)

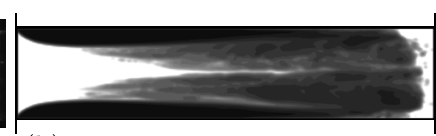

(h)

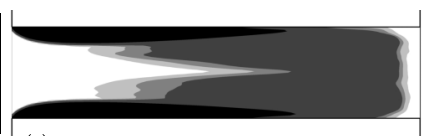

(i)

FIG. 6. Side view of vapor structures inside the nozzle for $\sigma=1.27$ (a)-(c), $\sigma=0.78$ (d)-(f), and $\sigma=0.65$ (g)-(i). Left column: instantaneous vapor structures observed in experiments [Reprinted with permission from A. Sou, S. Hosokawa, and A. Tomiyama, "Effects of cavitation in a nozzle on liquid jet atomization," Int. J. Heat Mass Transfer 50, 3575 (2007). Copyright 2007 Elsevier]. Middle column: contours of instantaneous, depth averaged vapor volume fraction $0.01<\langle\alpha\rangle_{z}<1.0$ in logarithmic scale. Right column: contours of time and depth averaged vapor fraction $\langle\langle\alpha\rangle\rangle_{z}=\{0.01,0.05,0.1\}$.

experiment (right column) in comparison with LES results for the depth-averaged instantaneous vapor volume fraction $\langle\alpha\rangle_{z}$ (middle column) and time- and depth-averaged vapor volume fraction $\langle\langle\alpha\rangle\rangle_{z}$ (right column).

For a high cavitation number, $\sigma=1.27$, no cavitation is observed in the experiment, see Fig. 6(a). The LES simulation, Figs. 6(b) and 6(c), predicts a small amount of vapor to be generated in the shear layer at the nozzle inlet. In contrast to the nozzle geometry in the experiment, which is assumed to exhibit a small, but unspecified radius due to manufacturing and hydrodynamic erosion, the numerical grid prescribes a perfectly sharp edge that promotes rupture of the liquid.

For moderate cavitation numbers, $\sigma=0.78$, cavitation is observed in the shear layer up to approximately $35 \%$ of the nozzle length, see Figs. 6(d)-6(f). In the simulation, these sheets show a periodic shedding, which is induced by the re-entrant jet near the wall. Furthermore, smaller, cavitating vortices driven by the shear layer detach and are convected downstream, where they collapse under the higher surrounding pressure. After very strong collapses, a subsequent rebound and evaporation of liquid due to the induced expansion wave is observed in the LES.

The lowest cavitation number under investigation, $\sigma=0.65$, is characterized by a supercavitation state and forms a stable vapor sheet, which spans from the nozzle inlet to the outlet. In contrast to the experiments, Fig. 6(g), the LES predicts vapor regimes in the center of the duct starting at approximately $50 \%$ of the nozzle length, see Figs. 6(h) and 6(i). This can be attributed to large, cavitating vortical structures, which are discussed in detail below.

Based on the time and depth averaged vapor volume fraction, we determine the non-dimensional cavitation length $L_{c}^{*}$, which we define as the maximum stream-wise extent of the 5\%-isocontour of the time- and depth-averaged void fraction with respect to the nozzle length. Figure 7 shows $L_{c}^{*}$ as a function of the Reynolds number (a) and of the cavitation number (b), together with corresponding experimental data. Our results are in very good agreement with the experiments by Sou et al. ${ }^{39}$

A quantitative comparison with LDV measurements, where available, is given in Figs. 8-10. The plots show $x$ and $y$ components of mean velocities $\langle u\rangle$ and $\langle v\rangle$, and velocity fluctuations $u^{\prime}$ and $v^{\prime}$ on the $z$-symmetry plane over the half-width of the duct, where $y=0$ corresponds to the nozzle wall and $y=2 \mathrm{~mm}$ to the centerline.

For a cavitation number of $\sigma=1.27$, see Fig. 8, mean streamwise velocity components show excellent agreement between LES and experiment. An analysis of the velocity fluctuations reveals a significantly lower turbulence intensity, in particular in the $v^{\prime}$ component, at the centerline of the incoming flow at $x=0.5 \mathrm{~mm}$, see Figs. 8(c) and 8(d). The high level of turbulence in the experimental data is assumed to be due to disturbances induced by the plunger pump system and by flow deflections and throttles upstream of the point of interest. Such effects cannot be accounted for in 

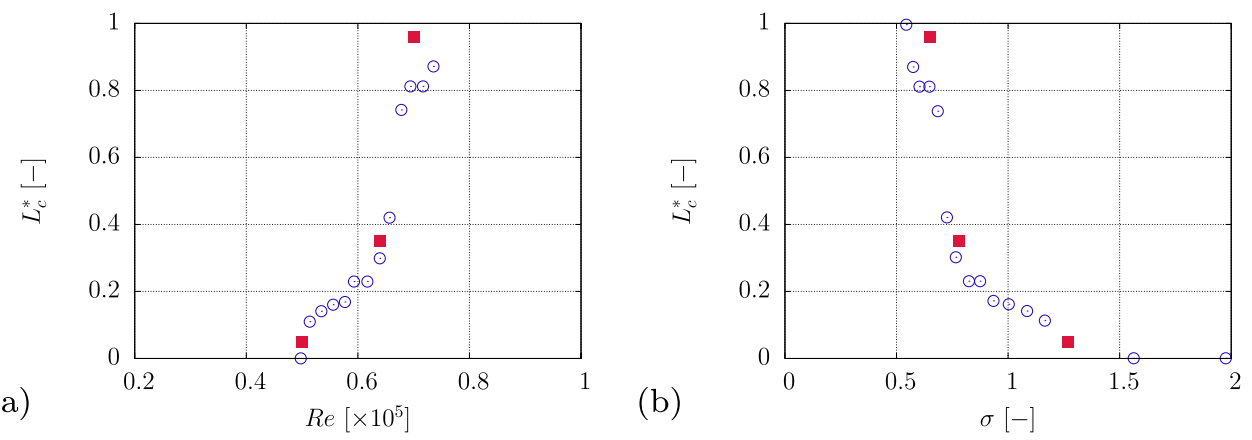

FIG. 7. Non-dimensional cavitation length $L_{c}^{*}$ vs. Reynolds number $\operatorname{Re}(a)$ and cavitation number $\sigma$ (b) from LES simulation (red filled square) and experiments ${ }^{39}$ (blue circle).

the LES. This is further elaborated in Appendix B, where effects of the inflow boundary conditions are excluded as a cause for this disagreement between simulation and experiment. Towards the nozzle outlet, the deviation of the velocity fluctuations in the core flow becomes smaller. At the edge of the shear layer, which is slightly wider in the LES, both simulation and experiment show peak values of the stream-wise turbulence intensity of approximately $30 \%$.

A good agreement is also found for a smaller cavitation number of $\sigma=0.78$, see Fig. 9. Again, the level of turbulent fluctuations is underestimated at the inlet but tends towards the experimental values in near-wall regions at the nozzle outlet, Figs. 9(c) and 9(d). LDV measurements in vapor regions are not possible. Hence, only the LES reveals the peak in the fluctuation quantities at the edge of the cavitating shear layer at $x=0.5 \mathrm{~mm}$, and the back-flow region with values of negative stream-wise velocity in the range of $0 \mathrm{~mm}<y<0.3 \mathrm{~mm}$ inside the detached vapor sheet.

The results for a cavitation number of $\sigma=0.65$ are shown in Fig. 9. In this case, the LES predicts larger water-vapor regions than the experiment in the downstream half section of the duct. Due to the smaller effective nozzle cross section, this leads to a higher mean stream-wise velocity

(a)
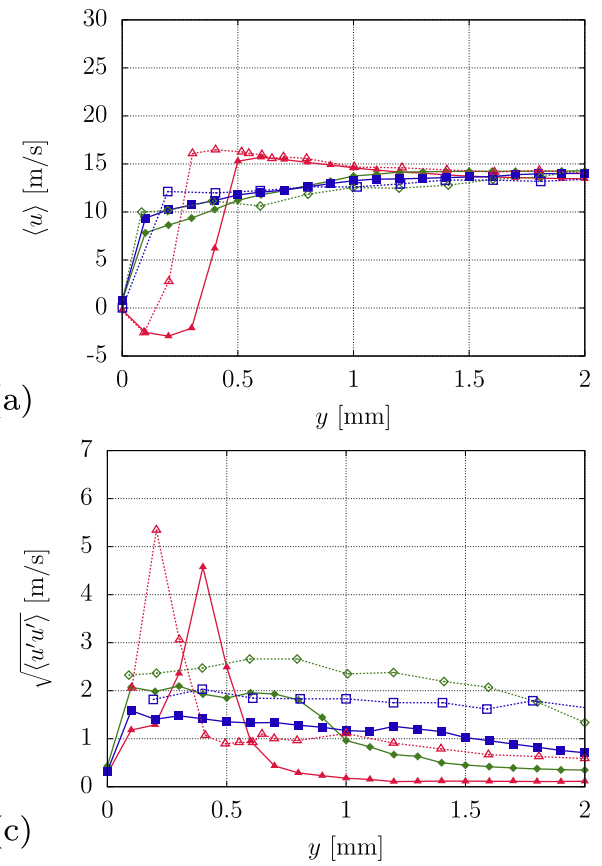

(b)

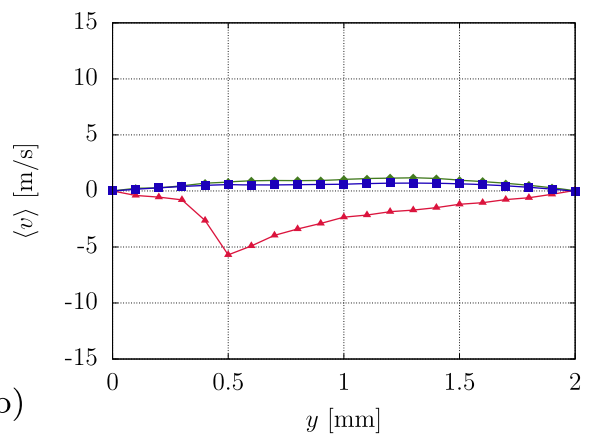

(d)

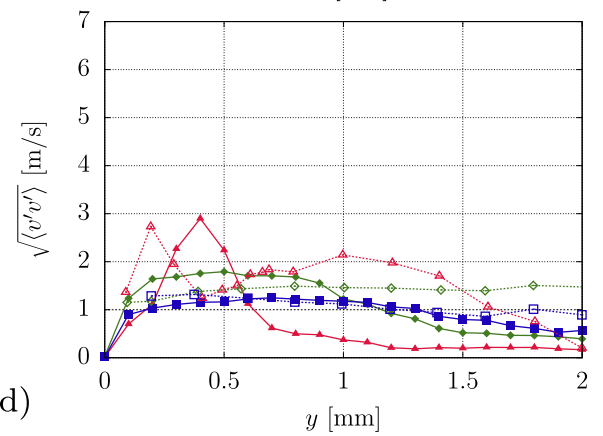

FIG. 8. First operating point $\sigma=1.27$ : Mean and fluctuating velocity components at positions $x=0.5 \mathrm{~mm}$ (red triangle), $x=8.0 \mathrm{~mm}$ (green diamond), and $x=13.0 \mathrm{~mm}$ (blue square); filled symbols/solid lines represent simulation results, empty symbols/dashed lines are experimental results. ${ }^{38,39}$ 
(a)
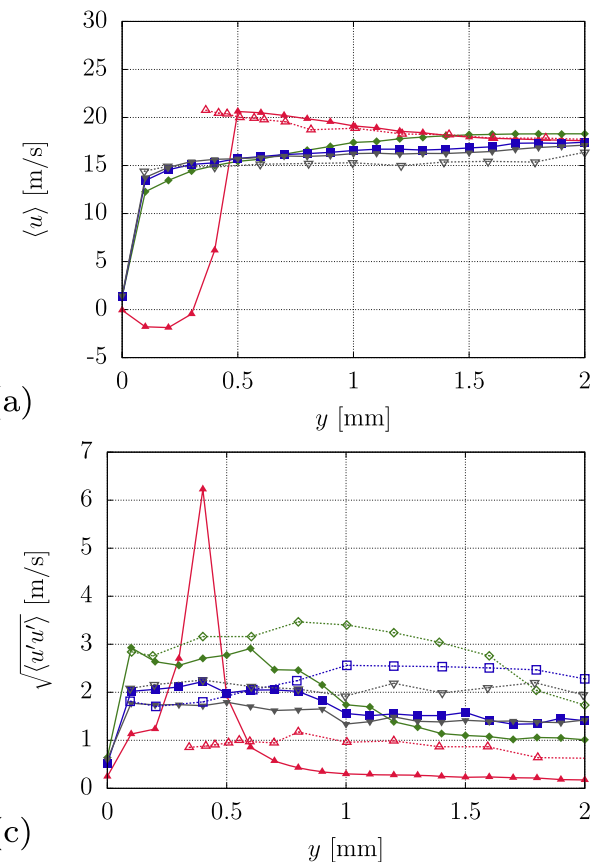

(b)
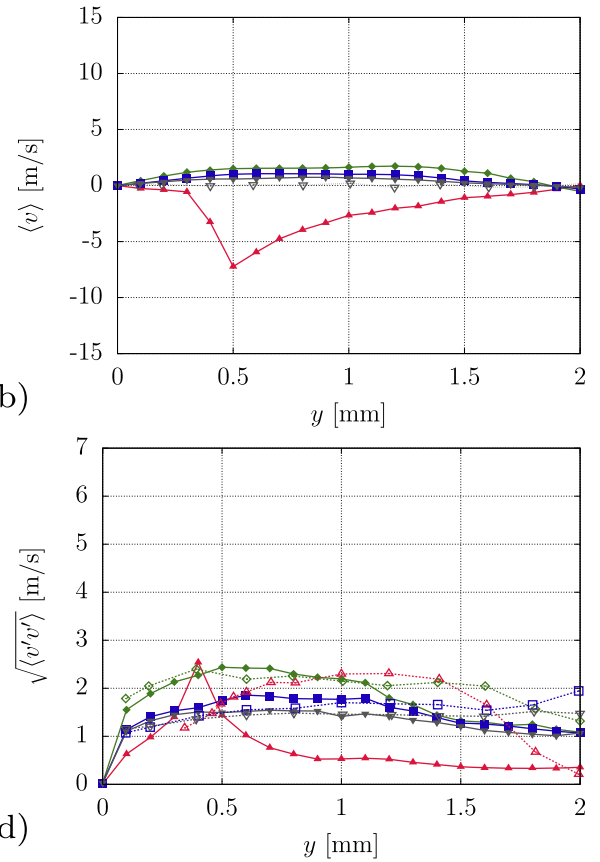

FIG. 9. Second operating point $\sigma=0.78$ : Mean and fluctuating velocity components at positions $x=0.5 \mathrm{~mm}$ (red triangle), $x=8.83 \mathrm{~mm}$ (green diamond), $x=13.0 \mathrm{~mm}$ (blue square), and $x=15.0 \mathrm{~mm}$ (gray inverse triangle); filled symbols/solid lines represent simulation results, empty symbols/dashed lines are experimental results. ${ }^{38,39}$

than measured in the experiment. The deviation of the mean velocity $\langle v\rangle$ in the range $0.3<y<0.5$ can likely be accredited to the slightly thicker boundary layer at this position due to the perfectly sharp inlet edge in the LES grid. Additionally, the vapor regions damp velocity fluctuations, see, e.g., Duke et al. ${ }^{35}$ and Egerer et al. ${ }^{32,65}$ Only after $x=15 \mathrm{~mm}$ a strong rise in turbulence intensity

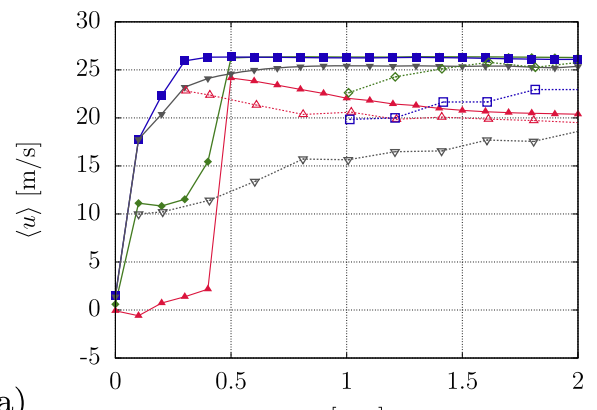

(a)

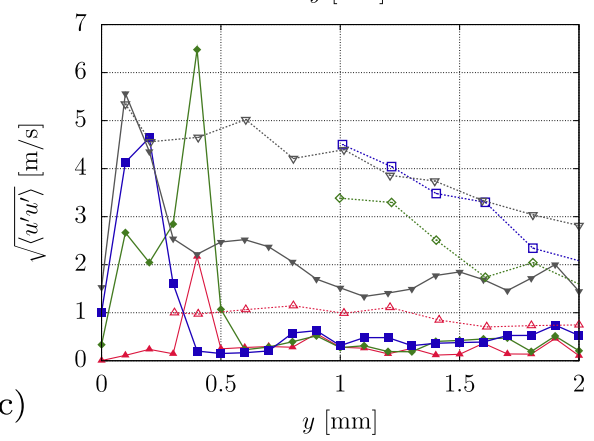

(b)
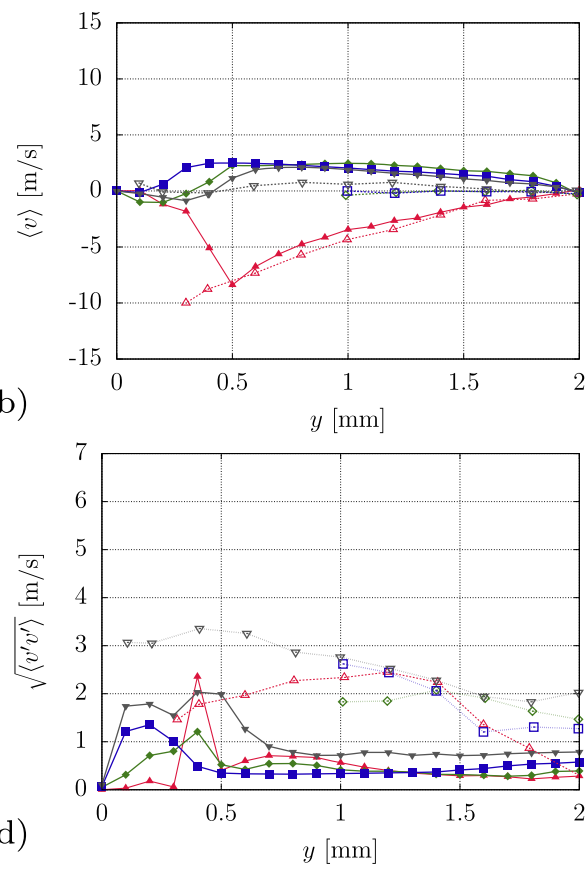

FIG. 10. Third operating point $\sigma=0.65$ : Mean and fluctuating velocity components at positions $x=0.5 \mathrm{~mm}$ (red triangle), $x=8.0 \mathrm{~mm}$ (green diamond), $x=13.0 \mathrm{~mm}$ (blue square), and $x=15.0 \mathrm{~mm}$ (gray inverse triangle); filled symbols/solid lines represent simulation results, empty symbols/dashed lines are experimental results. ${ }^{38,39}$ 
is observed when compared to velocity profiles located upstream of this position. This turbulence amplification is caused by collapse events at the end of the cavitation region and is further discussed below.

In summary, both a qualitative comparison in terms of position and size of vapor regions as well as a quantitative analysis of velocity fluctuations show good and reasonable, respectively, agreement between experiment and LES. For small cavitation numbers, the vapor regions are larger in the simulation, which causes damping of fluctuation quantities.

\section{B. Assessment of the three-dimensional cavitating nozzle flow}

In the following we analyze the three-dimensional flow field inside the nozzle. Figure 11 shows snapshots of iso-surfaces of coherent vortical structures visualized by the $\lambda_{2}$ criterion, ${ }^{66}$ together with cavitation regions and wall pressure.

For cavitation number $\sigma=1.27$, see Figs. 11(a) and 11(b), a transitional and eventually fully turbulent duct flow is recovered. Corner vortices, which originate from the boundary layers upstream of the nozzle, are stretched in stream-wise direction and quickly break up into small scale turbulence. This phenomenon has been studied extensively by Egerer et $_{\text {al }}{ }^{32}$

The highly unsteady shedding and collapse of cavitation structures in case of a cavitation number $\sigma=0.78$, Figs. 11(c) and 11(d), amplify turbulent fluctuations in the detached recirculation zone, which is identified by negative streamwise velocity. As a result, a fully turbulent flow is achieved earlier than in the non-cavitating case. The highly unsteady character of the flow at this operating point can also be noticed in the highly fluctuating wall pressure field, see Fig. 11(d), which shows the imprint of previously collapsed vapor clouds. Near the top wall of the nozzle, such a collapse event is captured in the snapshot (see marked position).

Results for a cavitation number of $\sigma=0.65$, Figs. 11(e) and 11(f), significantly differ from the higher cavitation numbers in terms of turbulent fluctuations and cavitation characteristics. For this operating point, stable corner vortices stretch up to approximately $60 \%$ of the nozzle length.
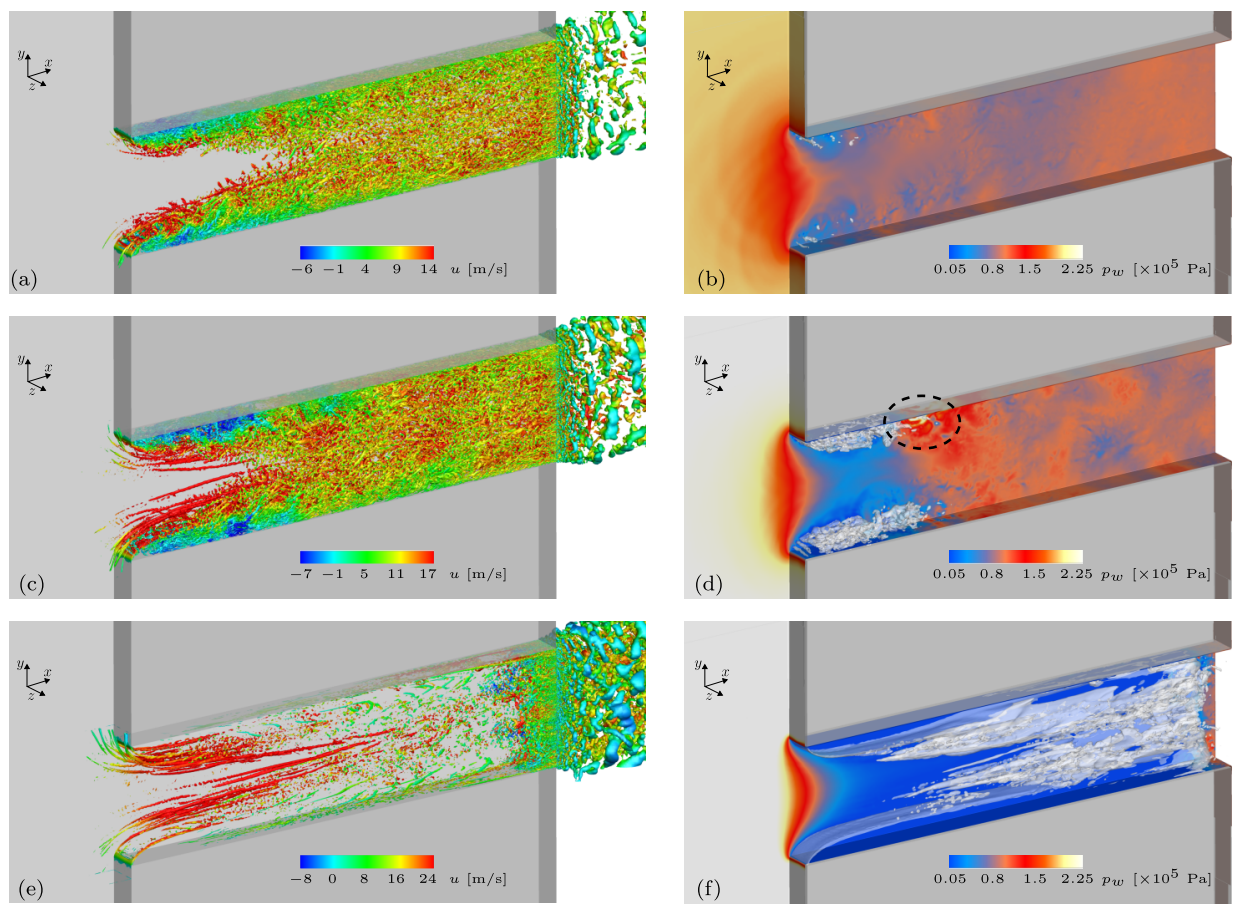

FIG. 11. Snapshot of iso-surfaces of $\lambda_{2}=-1 \times 10^{8} 1 / \mathrm{s}^{2}$ colored by streamwise velocity $u$ (left column) together with iso-surfaces of vapor volume fraction $\alpha=0.1$ and wall pressure (right column) for $\sigma=1.27, \sigma=0.78$, and $\sigma=0.65$ (from top to bottom). Black dashed marker shows an example for the wall pressure near a collapse event. 
These vortices start to cavitate at approximately $30 \%$ of the nozzle length and add to the stable cavitation sheet at the wall, which forms at the inlet edge. This observation is consistent with the work of Egerer et al. ${ }^{32}$ who found a similar, even more pronounced, behavior of cavitating corner vortices in their studies of cavitating micro-channels in agreement with reference experiments for a cavitation liquid throttle flow exiting into liquid. It is possible that these vortical structures become unstable in the experiment and break up due to the high level of turbulence, which would prevent the formation of stable vortex cavitation, or that geometrical uncertainties, which are not quantified in the description of the experimental setup, affect the nominal parameters at the low cavitation number case. Turbulent fluctuations are damped inside the vapor region and are strongly amplified at the nozzle outlet. This is in agreement with the findings of Dittakavi and Chunekar. ${ }^{67}$

\section{Effect of cavitating nozzle flow on jet break-up}

We now discuss the effect of the cavitating nozzle flow onto the liquid jet. Snapshots of our LES data are put side by side with experimental images in Fig. 12. The jet in the simulation is visualized by a $99 \%$-volume fraction of air. Data are extracted for a range of $16 \times 10^{-3} \mathrm{~m}<x<$ $32 \times 10^{-3} \mathrm{~m}$ to match the image selection shown by Sou et al. ${ }^{38,39}$

The experimental images show a very similar jet structure for cavitation numbers $\sigma=1.27$ and $\sigma=0.78$, see Figs. 12(a) and 12(d). For $\sigma=0.65$, Fig. 12(g), in contrast, spray formation is observed. Small droplets and ligaments of liquid detach from the surface and cause an increased jet angle.

Our numerical results, see Figs. 12(b), 12(e), and 12(h), show only little effect of the cavitation number on the average jet angle in the $x$ - $y$ plane. Rather than spray formation, we observe only a slightly more disturbed jet surface structure at the lowest cavitation number. However, significant differences between the higher cavitation numbers $\sigma=1.27$ and $\sigma=0.78$, and the low cavitation number $\sigma=0.65$ are observed in the $x$ - $z$ plane, see Figs. 12(c), 12(f), and 12(i). We clearly notice a widening of the jet and a detachment of large and few small liquid structures from its surface, which closely resembles the observations in the experiments of Sou et al ${ }^{38,39}$ This effect of the cavitation number on the jet spreading angle is more clearly visible in the time-averaged data, see Fig. 13.

The primary break-up of a liquid jet, which is driven by inertia, can be divided into two stages. Initial perturbations of the jet surface are triggered near the nozzle exit. These perturbations are amplified in the liquid-gas shear layer under the influence of aerodynamic forces, resulting in secondary jet break-up in which the formation of droplets and ligaments dominates. For the initial
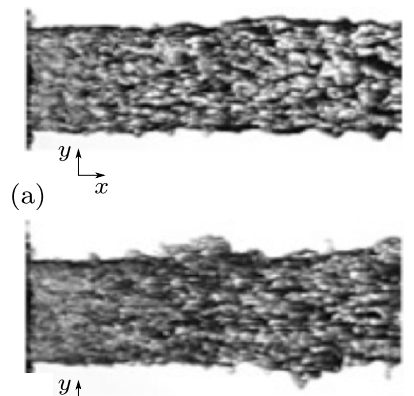

(d) $\stackrel{y \uparrow}{\longrightarrow}$

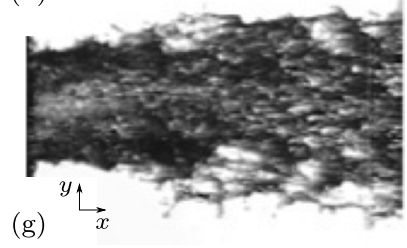

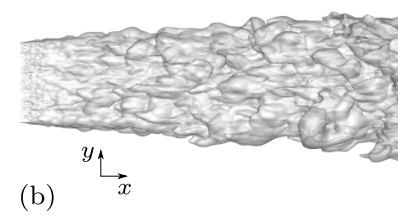
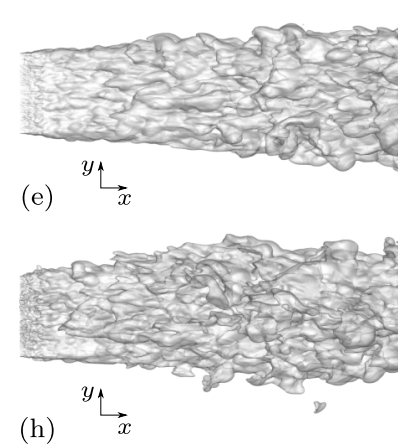

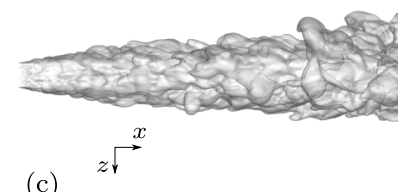

(c)

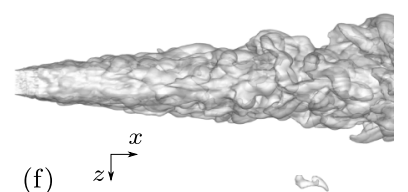

(f)

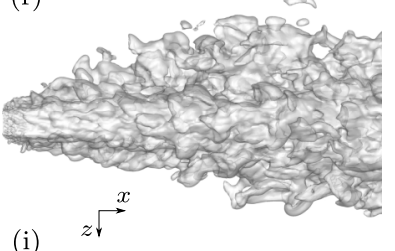

FIG. 12. Experimental transmitted light images (left column, reprinted with permission from A. Sou, S. Hosokawa, and A. Tomiyama, "Effects of cavitation in a nozzle on liquid jet atomization," Int. J. Heat Mass Transfer 50, 3575 (2007). Copyright 2007 Elsevier) and LES snapshots of $x-y$ view (middle column) and $x-z$ view (right column) showing iso-surfaces of gas volume fraction $\beta_{G}=0.99$ in the range $16 \mathrm{~mm}<x<32 \mathrm{~mm}$ for $\sigma=1.27, \sigma=0.78$, and $\sigma=0.65$ (from top to bottom). 


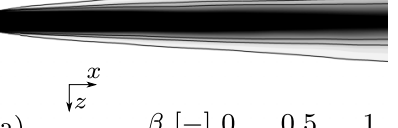

(a) $\beta[-] 0 \quad 0.5 \quad 1$

(b)

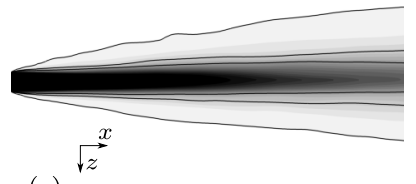

(c)

FIG. 13. Time-averaged gas volume fraction $\beta_{G}$ on the $y$-symmetry plane in the range $16 \mathrm{~mm}<x<32 \mathrm{~mm}$ for $\sigma=1.27$, $\sigma=0.78$, and $\sigma=0.65$ (from left to right). Black lines show contour levels of $\beta_{G}=\{0.8,0.9,0.99\}$.

stage, momentum generation towards the liquid-gas interface is of main importance. In addition to initial disturbance of the liquid-air interface due to Kelvin-Helmholtz (KH) type instabilities, we identify three main mechanisms from an analysis of our simulation data that lead to distortions of the jet surface and hence to a widening and break-up of the jet: turbulence production in terms of velocity fluctuations inside the nozzle near the outlet region, entrainment of non-condensable free gas, which enters the nozzle region from the outlet volume, and collapse events inside the emerging liquid jet. In the following, each of these mechanisms is briefly discussed.

\section{Kelvin-Helmholtz instabilities}

Liquid jet and gas phase form a shear layer at the interface in the outflow region, which quickly becomes unstable to small disturbances and forms large-scale $\mathrm{KH}$ vortical structures, as has been discussed extensively in the literature. ${ }^{68-70}$ These formations grow until the aerodynamic forces due to the velocity difference with respect to the quiescent surrounding gas break off large structures, which eventually lead to Rayleigh break-up and droplet formation. ${ }^{43}$ In Fig. 14 , KH vortical structures are visualized by contours of the $z$-vorticity magnitude $\omega_{z}$ in the $x y$-symmetry plane (top row) and of the $y$-vorticity magnitude $\omega_{y}$ in the $x z$-symmetry plane. The jet surface is highlighted by an iso-line of gas volume fraction $\beta_{G}=0.99$.

For the two higher cavitation numbers, $\sigma=1.27$ and $\sigma=0.78$, only low values of vorticity production are found at the liquid gas interface. For a low cavitation number, $\sigma=0.65$, large $\mathrm{KH}$ vortices are clearly visible, which detach from the jet.

\section{Effect of turbulent fluctuations}

Sou et al. ${ }^{38,39}$ argue that strong turbulent fluctuations induced by collapses of vapor regimes just upstream of the nozzle exit induce additional momentum towards the liquid-gas interface and hence may cause the increase in spray angle and, ultimately, jet atomization observed in their experiments.

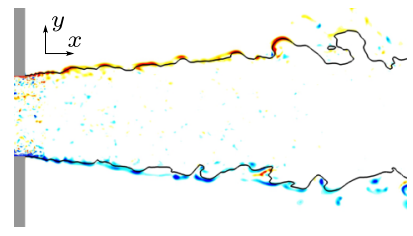

(a)

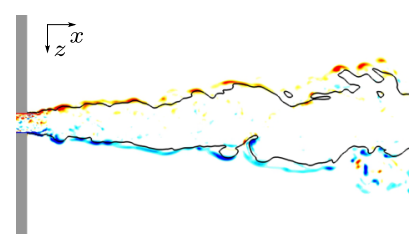

(d)

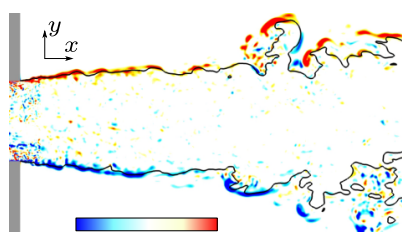

(b) $-40 \quad 0 \quad 40 \omega_{z}\left[\times 10^{4} 1 / s\right]$

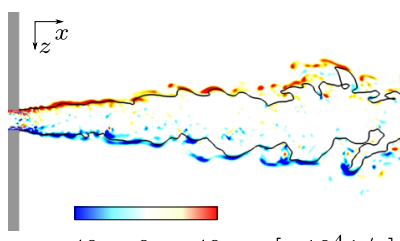

(e) $\quad-40 \quad 0 \quad 40 \omega_{y}\left[\times 10^{4} 1 / s\right]$

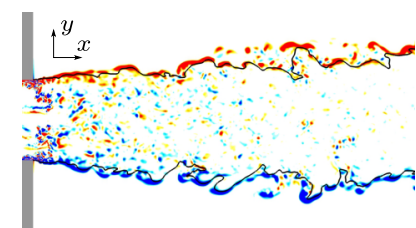

(c)

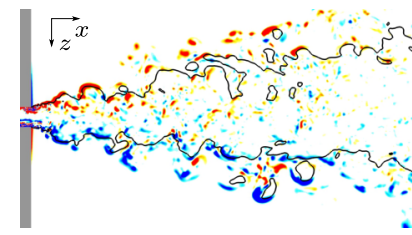

(f)

FIG. 14. Contours of $z$-vorticity magnitude $\omega_{z}$ in the $x y$-symmetry plane (top row) and $y$-vorticity magnitude $\omega_{y}$ in the $x z$-symmetry plane (bottom row) together with iso-contour for gas volume fraction $\beta_{G}=0.99$ (black line) for $\sigma=1.27$, $\sigma=0.78$, and $\sigma=0.65$ (from left to right). 

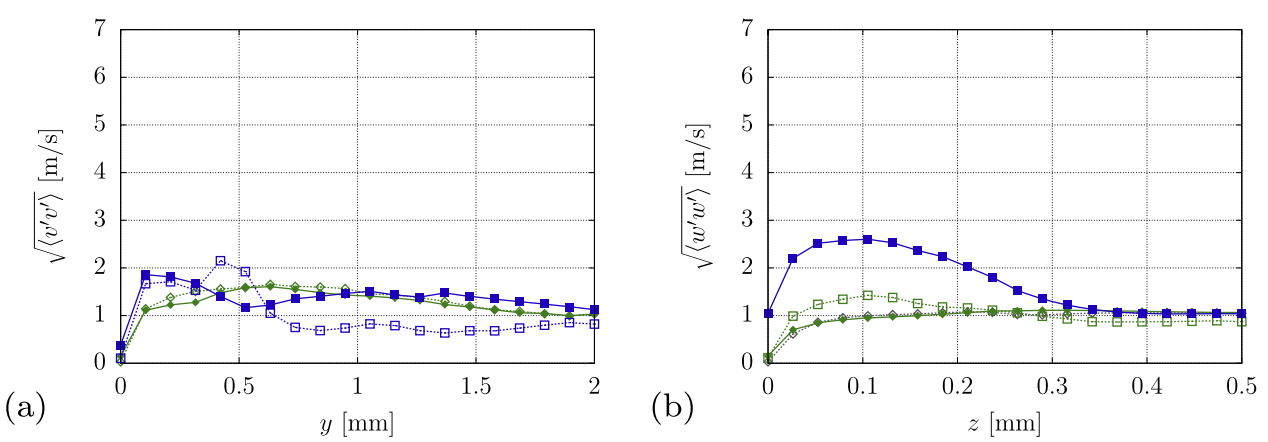

FIG. 15. Components of wall-normal velocity fluctuations in $y$ - and $z$-direction at the nozzle outlet at $x=15.0 \mathrm{~mm}$ (empty symbols/dashed lines) and $x=16.0 \mathrm{~mm}$ (filled symbols/solid lines) for $\sigma=0.78$ (green diamond) and $\sigma=0.65$ (blue square).

The collapse of single bubbles and bubble clouds interacts with the surrounding flow field on multiple scales. Dabiri et al. ${ }^{71}$ observe that vorticity is generated at the surface of a single bubble in a shear flow during collapse, which could add to larger vortical structures in the liquid. This effect, however, cannot be resolved in our LES. We suspect that the observed increase of the small scale fluctuations on the turbulence level is caused by micro-jetting during the collapse process of aspherical vapor structures and vapor clouds, which creates a strong increase of local kinetic energy, see Adams and Schmidt. ${ }^{72}$ Dittakavi and Chunekar ${ }^{67}$ moreover found that the collapse of vapor structures causes a substantial increase in the baroclinic torque. In addition, turbulent fluctuations are amplified when processed by shock waves, see, e.g., Larsson et al. ${ }^{73}$ and Hickel et al. ${ }^{60}$ In a cavitating cloud collapse, this effect can be intensified by chain reactions of collapsing cavity arrays, as found by Lauer et al. ${ }^{21}$

Figure 15 shows the wall-normal velocity fluctuations in $y$ - and $z$-direction at the outlet extracted from our simulations. $y=0$ and $z=0$ correspond to the wall coordinate. In the LES, only a small increase in wall-normal velocity fluctuations, Fig. 15(a), is detected in the proximity of the top wall near the nozzle exit when comparing $\sigma=0.78$ with $\sigma=0.65$. In contrast, in $z$-direction, see Fig. 15(b), a significant increase in the $w^{\prime}$ fluctuation magnitude is found for the supercavitating
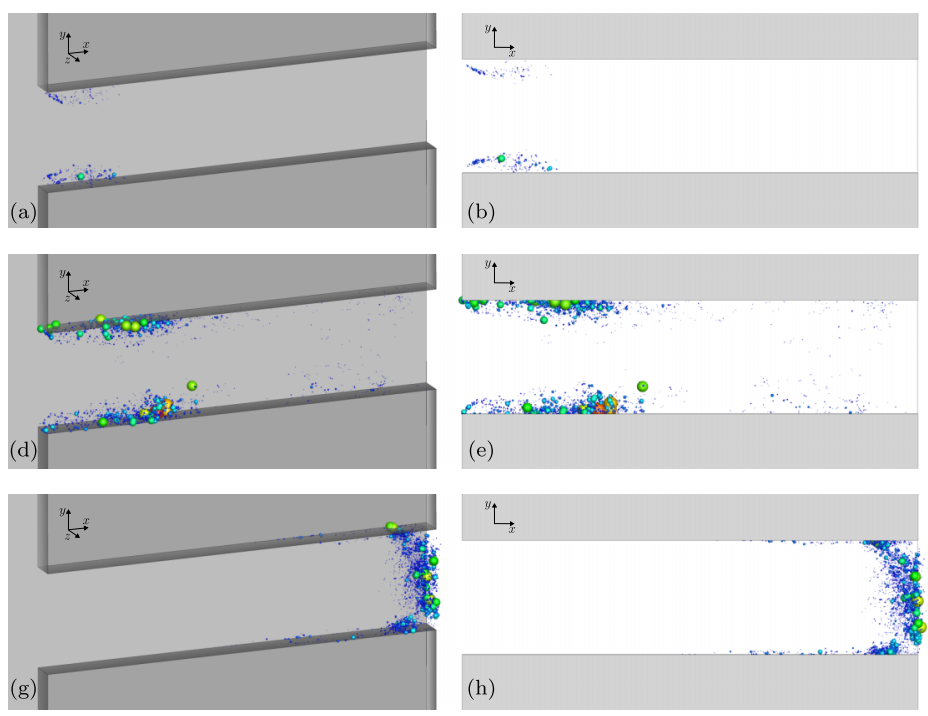
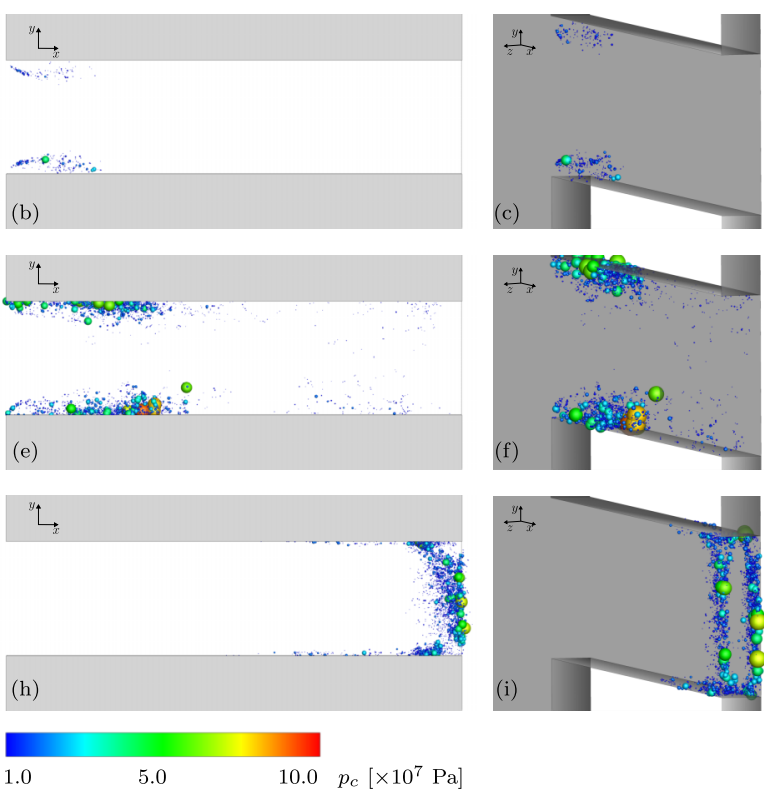

FIG. 16. Spatial distribution of isolated collapses detected during an analysis interval of $\Delta t=2 \mathrm{~ms}$ for $\sigma=1.27, \sigma=0.78$, and $\sigma=0.65$ (from top to bottom). Size and color of each sphere is based on the normalized collapse pressure $p_{c}$. Collapse events with a collapse pressure $p_{c}<30 \times 10^{5} \mathrm{~Pa}$ are not shown. 

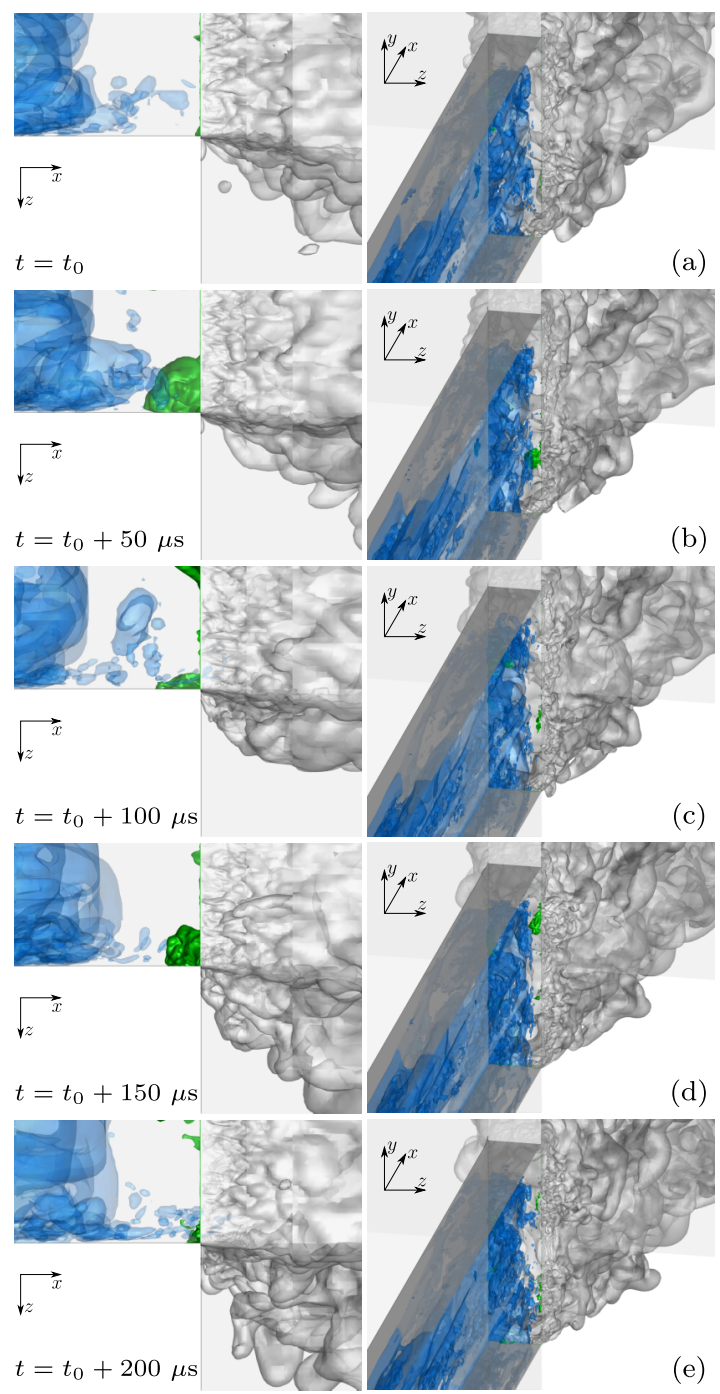

(c)
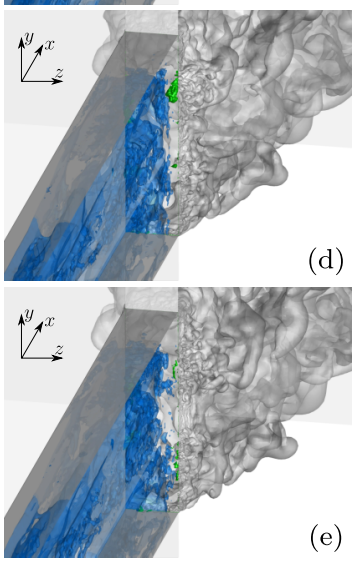

FIG. 17. Visualization of gas entrainment process into the nozzle (left column: top view; right column: perspective view). Snapshots show iso-surfaces of $\alpha=0.1$ (blue), together with gas volume fraction $\beta_{G}=0.99$ (gray) and entrained gas volume fraction $\beta_{G}=0.1$ (green).

nozzle with $\sigma=0.65$. The maximum amplitude of the $w^{\prime}$ fluctuations for $\sigma=0.65$ is approximately 2.5 times higher than for $\sigma=0.78$.

In our numerical simulation, we detect collapse events with an algorithm developed by Mihatsch et al. ${ }^{74}$ which considers the re-condensation of vapor volume content inside a computational cell together with an analysis of the local velocity divergence. The normalized peak collapse pressure $p_{c}$ is obtained by scaling the observed maximum $p_{m}$,

$$
p_{c}=p_{m} \frac{V_{\Omega}^{1 / 3}}{l_{\text {ref }}},
$$

where we set the reference length scale $l_{r e f}=3.75 \times 10^{-6} \mathrm{~m}$ to compensate for the effect of grid resolution onto the collapse pressure inside the cell with volume $V_{\Omega}$. Figure 16 shows the position of detected collapse events during a time interval of $\Delta t=2 \mathrm{~ms}$. Each event is represented by a sphere, whose size and color scale with normalized collapse pressure $p_{c}$. In case of $\sigma=1.27$, see Figs. 16(a)-16(c), only few weak events are detected near the nozzle inlet. For $\sigma=0.78$, Figs. 16(d)-16(f), the location of collapse events is restricted approximately to the first $30 \%$ of the duct near the upper and lower walls. Only few collapses, which are mainly caused by rebounding 


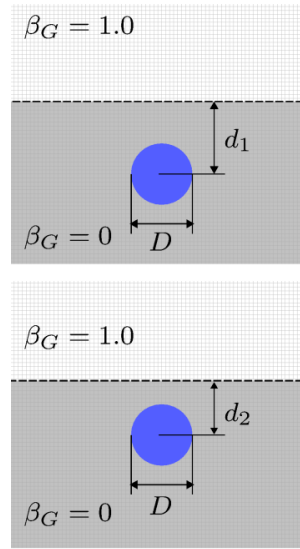

(a) $t=t_{0}$
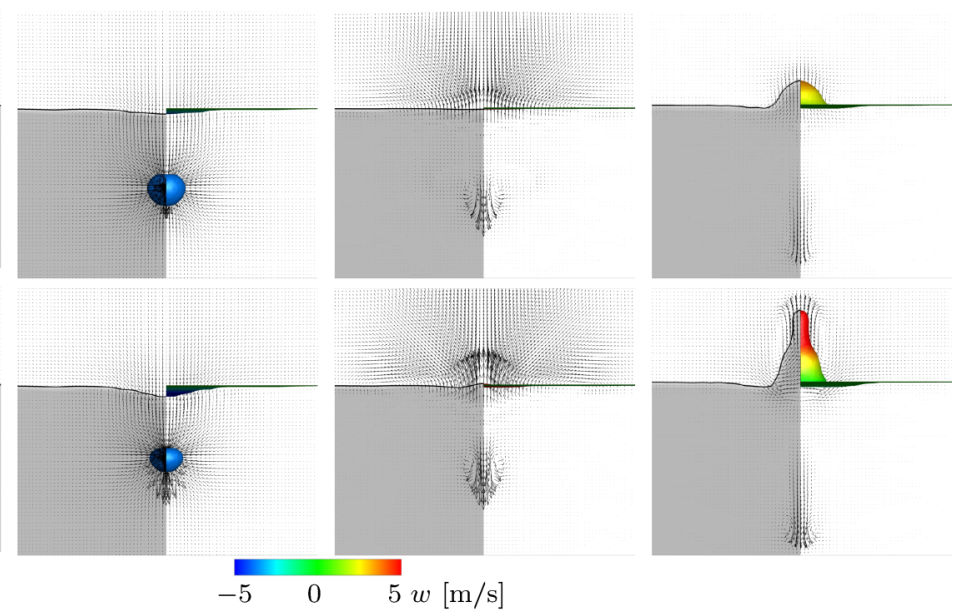

(c) $t=t_{0}+9.0 \mu \mathrm{s}$

(d) $t=t_{0}+27.0 \mu \mathrm{s}$

FIG. 18. Collapse of single cavitation bubbles in the proximity of a liquid-gas interface at various instants in time. Bubble has an initial diameter $D=0.2 \mathrm{~mm}$ and is placed at a distance $d=\{0.17,0.145\} \mathrm{mm}$ from the interface. Snapshots show iso-surfaces of vapor volume fraction $\alpha=0.1$ (blue), gas volume fraction $\beta_{G}=0.99$ colored by interface-normal velocity $w$ (right half of figures), and liquid mass fraction on the symmetry plane (shaded grey) together with contour-line $\beta_{G}=0.99$ (left half of figures).

vapor bubbles, are detected downstream of this region. In the supercavitating case, $\sigma=0.65$, the majority of collapse events is detected near the nozzle outlet, see Figs. 16(g)-16(i). In addition to events at the upper and lower duct walls at approximately $90 \%$ of the nozzle length, strong events are recorded in the LES at the side walls directly at the outlet edge. The events in this region are caused by the collapse of vapor structures near the side walls predicted in our LES, which are not present in the experiment. The position of the collapse events coincides with the location of amplification of turbulent fluctuations, which confirms the hypothesis of Sou et al. ${ }^{38,39}$

\section{Effect of gas entrainment}

Gas entrainment into the nozzle is found to introduce large disturbances to the jet, which leads to an increase of the average jet angle. Low pressure vapor regions, which are present just upstream of the nozzle outlet and extend to the exit at the side-walls, cause a pressure gradient directed from the nozzle into the gas filled plenum. The initial phase of the collapse process of a cavitation region furthermore causes an acceleration of surrounding fluid towards the center of the structure. Near the nozzle outlet, such events cause gas to enter the duct region.

This mechanism is depicted as a series of snapshots in Fig. 17. Gas that has passed the exit plane in upstream direction is visualized though green iso-surfaces of $\beta_{G}=0.1$. At time $t=t_{0}$, Fig. 17(a), a large vapor structure reaches the nozzle outlet. As soon as the large structure collapses, gas is sucked into the nozzle, see Fig. 17(b). When the gas is pushed back by the liquid, Fig. 17(c), a significant acceleration of water in $z$-direction is observed, which results in a larger angle of the jet at the outlet. Few instants later in time, see Figs. 17(d) and 17(e), the same process is observed in the upper nozzle region.

\section{Effect of collapse events inside the jet}

An potential additional mechanism promoting jet break-up is suspected in the collapse of vapor bubbles near a liquid-gas interface. This phenomenon was, e.g., discussed by Robinson et al. ${ }^{75}$ and Obreschkow et al. ${ }^{76}$ who describe the interaction of collapsing cavitation bubbles with a free planar and curved surfaces. The authors demonstrate that a bubble collapse near a liquid-gas interface creates a primary liquid jet pointing away from the interface and a secondary jet towards the interface. The latter causes a liquid jet emerging from the surface. The intensity of this mechanism depends on the size of the cavitation bubble and its distance from the interface. 


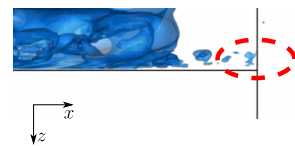

(a) $t=t_{0}$

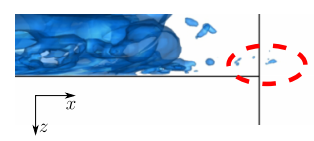

(b) $t=t_{0}+7.5 \mu \mathrm{s}$

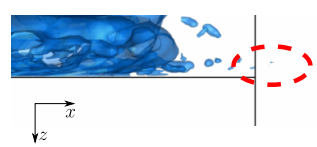

(c) $t=t_{0}+10.0 \mu \mathrm{s}$

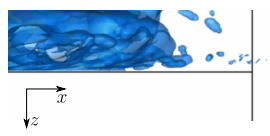

(d) $t=t_{0}+12.5 \mu \mathrm{s}$

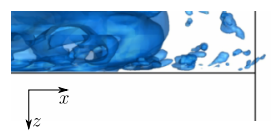

(e) $t=t_{0}+22.5 \mu \mathrm{s}$
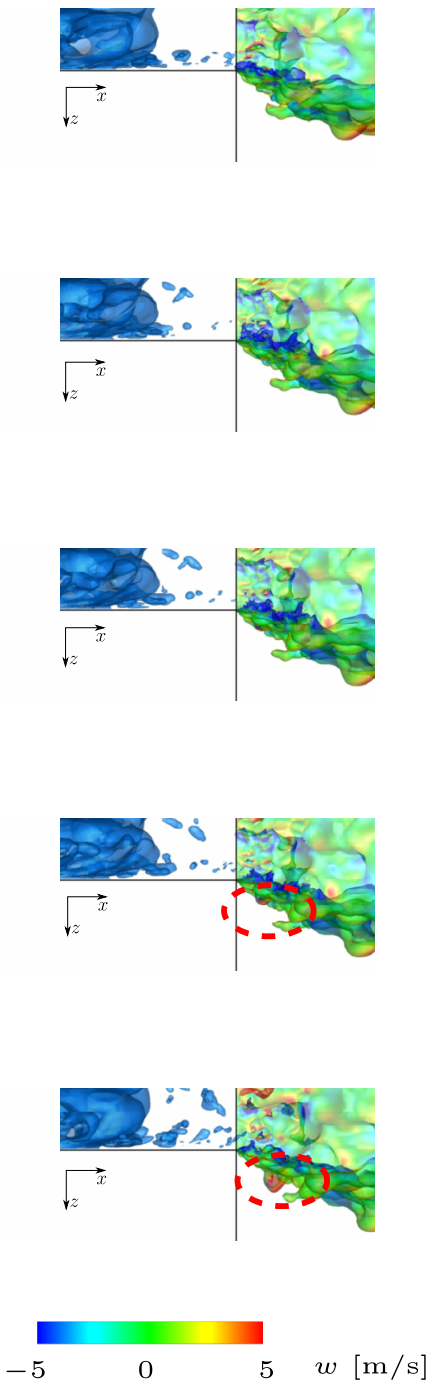
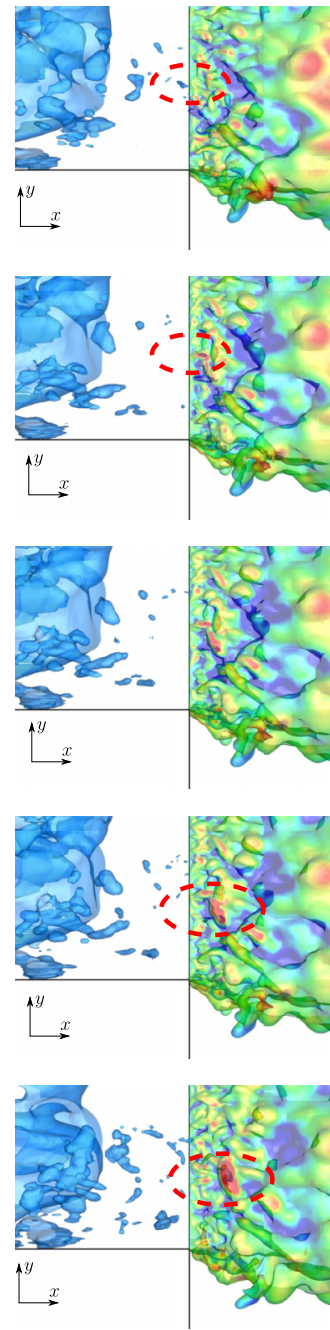

FIG. 19. Collapse event inside the jet. Snapshots show iso-surfaces of $\alpha=0.1$ (blue) and gas volume fraction $\beta_{G}=0.99$ colored by $w$ velocity. Snapshots are taken at the same instant in time. Collapse position and emerging liquid jet are highlighted by a red-dashed marker.

To demonstrate the ability of our model to represent this phenomenon, we have conducted a simple simulation to investigate this mechanism, as shown in Fig. 18. We initialize the domain with a liquid-gas interface at $p=1 \mathrm{~atm}$ and place a vapor bubble with $\alpha_{v}=99 \%$ inside the liquid. The bubble has a diameter of $D=0.2 \mathrm{~mm}$ and is resolved with approximately 16 cells across the bubble diameter on a homogeneous grid. We vary the distance $d$ between the bubble center and the interface, see Fig. 18(a).

Snapshots show iso-surfaces of vapor volume fraction, gas volume fraction, and liquid mass fraction (right half of figures) together with contour-line $\beta_{G}=0.99$ on the symmetry plane (left half of figures). The liquid-gas interface is colored by interface-normal velocity $w$. The bubble collapses under the high surrounding pressure, which is shown as a time series in Figs. 18(b)-18(d). As soon as the bubble has collapsed, Fig. 18(c), which causes the liquid-gas interface to contract towards the collapse center, the formation of two jets is visible. The primary jet is directed away from the liquid-gas interface. The secondary jet, as found by experimental studies, points towards the interface and causes a small jet filled with water to be ejected into the gas domain, Fig. 18(c). The intensity of this jet, as well as inherent time scales, depends on the initial bubble diameter and its distance from the interface. The simplified study shows that effect of the secondary liquid 
jet penetrating the interface can be captured without surface tension in the early stages, before the formation of droplets and ligaments dominates the process, which cannot be captured by our current model.

A similar phenomenon, which we suspect to be based on the mechanism of a single bubble collapse near a liquid-gas interface as discussed above, is observed when cavitation regions pass the nozzle outlet and collapse inside the liquid jet near the liquid-gas interface. A confirmation of the occurrence of collapse events beyond the nozzle exit plane $(x>16 \mathrm{~mm})$ in case of a supercavitating flow is found in Fig. 16(h). A time series of what we suspect to be the footprint of a vapor collapse near the jet surface is presented in Fig. 19. We show iso-surfaces of vapor volume fraction $\alpha=0.1$ (blue) in a top view without a visualization of the jet (left column), together with top (middle column) and side views (right column) of vapor structures and an iso-surface of the gas volume fraction $\beta_{G}=0.99$ colored by $w$-velocity.

We follow a cavitation structure marked in Fig. 19(a). The structure is convected downstream and has passed the nozzle exit plane in Fig. 19(b). A short time after the vapor cloud has collapsed under the high pressure that is imposed from the surrounding fluid, see Fig. 19(c). A collapse near the jet surface causes an acceleration of liquid towards the liquid-gas interface and a small, high velocity liquid spike emerging from the jet surface, which are marked in Figs. 19(d) and 19(e).

In summary, the described mechanisms significantly add to the generation of momentum directed towards the liquid-gas interface, which causes a widening and primary break-up of the liquid jet surface.

\section{SUMMARY AND CONCLUSIONS}

Many experimental studies show that cavitation phenomena play a crucial role in jet break-up and vaporization. Still, the simulation of cavitating nozzle flows injected into a free gas phase remains a challenging task. As many complex models are limited to relatively simple configurations, one has to find a compromise between feasibility and applicability to realistic problems. We have extended a thermodynamic equilibrium cavitation model by a non-condensable gas component to formulate a simple, but highly effective approach for LES. Focus of the present study is the initial stage of the jet break-up. The simulation of the secondary break-up, that is, ligament and droplet formation, has not been considered since the current model does not include surface tension effects.

The model has been applied to a cavitating nozzle slot and jet flow injected into air. The setup of the present study has been subject to extensive experimental investigation. We have performed LES for three different cavitation numbers, each of which shows different characteristics of the nozzle flow and of the liquid jet. Cavitation structures within the duct resemble experimental results. Quantitative measurements of mean and fluctuating velocity components reveal a lower level of turbulence in the simulation, which we attributed to disturbances induced by the experimental apparatus. Other experiments for generic throttles, where cavitating liquid is ejected into liquid instead of air, have been reproduced successfully in Egerer $e t$ al..$^{32}$ The geometry is very similar to that of the current experiment, and it is plausible to expect that the flow in the inflow-chamber and throttle would exhibit similar flow structures. This applies in particular to the choked-nozzle case, where the nozzle flow to some extent is decoupled from outflow-chamber details. Secondary-flow vortices, which also have been observed in the experimental reference of Egerer $\mathrm{et} \mathrm{al.},{ }^{32}$ are not observed in the current reference experiment. The lack of secondary-flow structures could be caused by a deviation of the effective experimental cavitation number from the nominal one, which is used in the simulations.

In contrast to the experiments, we have observed that cavitation promotes the break-up of the jet in lateral direction. In addition to classical Kelvin-Helmholtz type large scale instabilities of the jet surface, three main mechanisms suggested to be responsible for jet break-up in cavitating liquid flows have been reproduced and analyzed, namely, turbulent fluctuations induced by the collapse of cavitation structures in the proximity of the exit plane of the nozzle, entrainment of gas into the nozzle, and collapse events inside the jet near the liquid-gas interface. All three mechanisms induce momentum directed away from the jet axis and lead to primary break-up of the jet. Collapse events near the exit and outside the nozzle region were found to be of particular importance. A fully compressible description of the flow is essential to capture such mechanisms. 
The model offers a straightforward way for incorporating further extensions, such as the solution of gas into the liquid and outgassing effects by adding a source term to the gas mass fraction transport equation. This may especially have an effect on cavitation collapse characteristics and, consequently, on the liquid jet when air is entrained into the nozzle. In addition, more complex thermodynamic descriptions for liquid and gas phases, such as water based on a Tait equation or diesel fuel for the investigation of realistic fuel injection systems, can be included and are subject to current investigations.

\section{ACKNOWLEDGMENTS}

Computing time was granted by the Leibnitz Supercomputing Centre (LRZ) of the Bavarian Academy of Sciences and Humanities. We would like to thank C. Egerer for sharing his expertise in the analysis of cavitating nozzle flows.

\section{APPENDIX A: DISCUSSION OF GRID CONVERGENCE BEHAVIOR}

To demonstrate the grid convergence behavior, we present simulation results for the developing cavitation number $\sigma=0.78$. As discussed above, we apply 5 different grids, which range
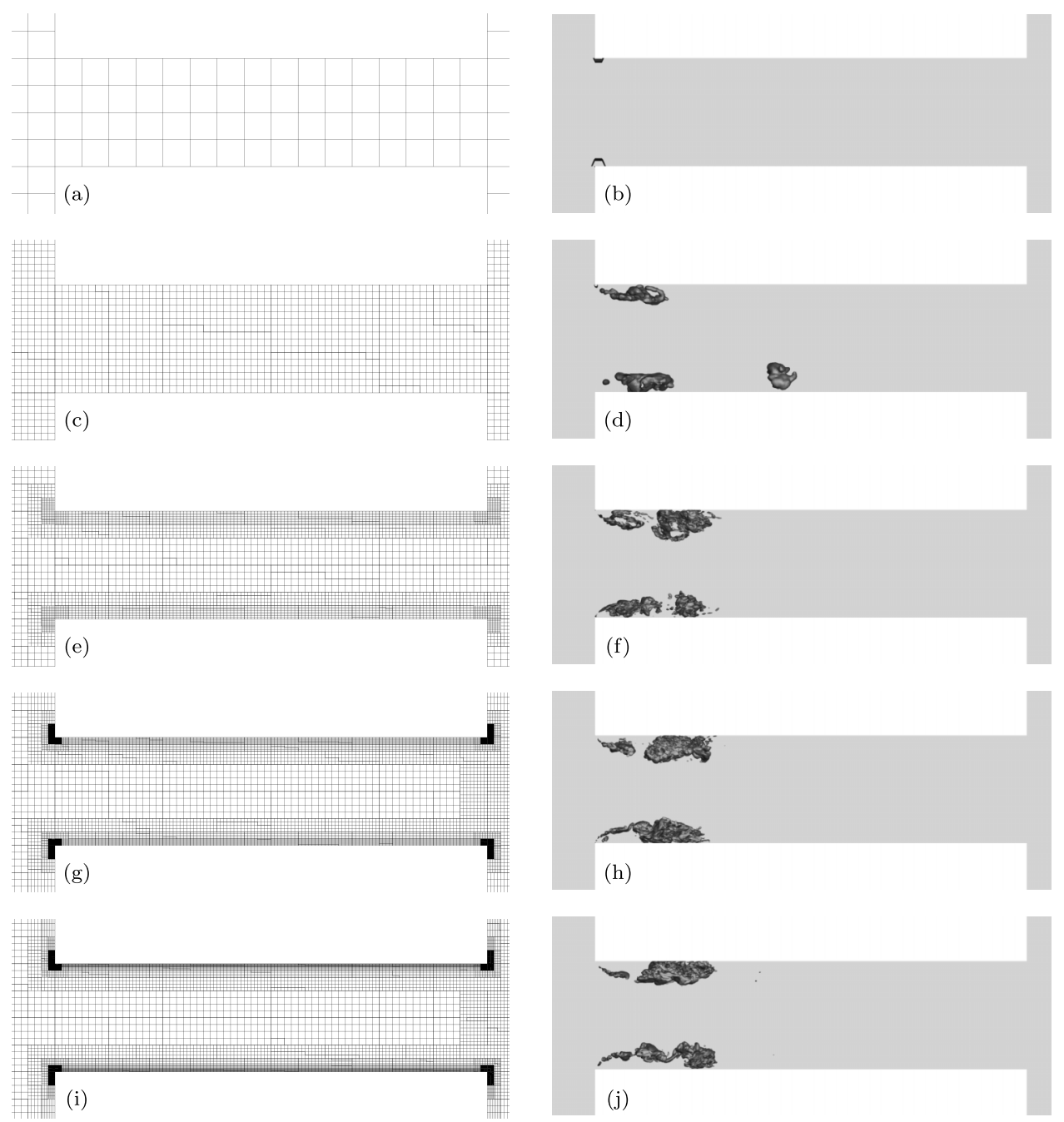

FIG. 20. Computational mesh for all applied grid levels 1-5 (from top to bottom): $x$ - $y$ view of the mesh in the $z$ symmetry plane (left column, only every fourth grid line is shown); instantaneous iso-surfaces of $10 \%$-void fraction (right column). 


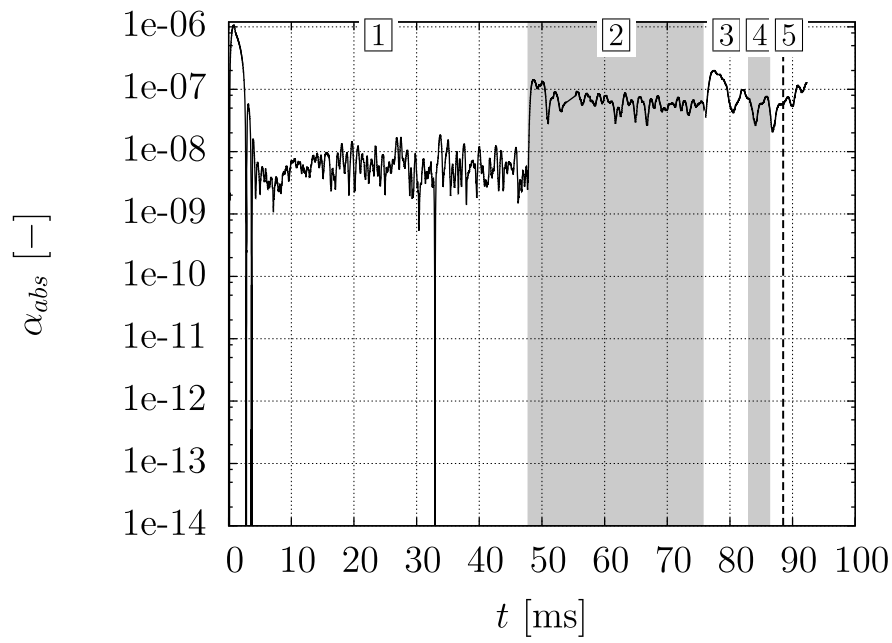

FIG. 21. Temporal evolution of the global vapor volume fraction $\alpha_{a b s}$ for $\sigma=0.78$. Grid levels are marked with numbers; sampling on the finest grid is started at the position marked with a dashed line.

from a very coarse grid, three intermediate grids, to the final fine grid. We initiate our simulation on the coarse grid (level 1). We then interpolate the solution to the intermediate grids (levels 2-4) and, finally, the fine grid (level 5) as soon as a steady behavior of the flow is found. Figure 20 shows the grid in the nozzle region (left column) along with instantaneous iso-surfaces of $10 \%$-vapor-volume fraction (right column) for the coarse grid, Figs. 20(a) and 20(b), the intermediate level 2, Figs. 20(c) and 20(d), level 3, Figs. 20(e) and 20(f), and level 4, Figs. 20(g) and 20(h), grids, as well as the fine grid, Figs. 20(i) and 20(j). Additionally, Fig. 21 shows the time evolution of the global vapor volume fraction. Grid levels are marked with the corresponding numbers.

(a)
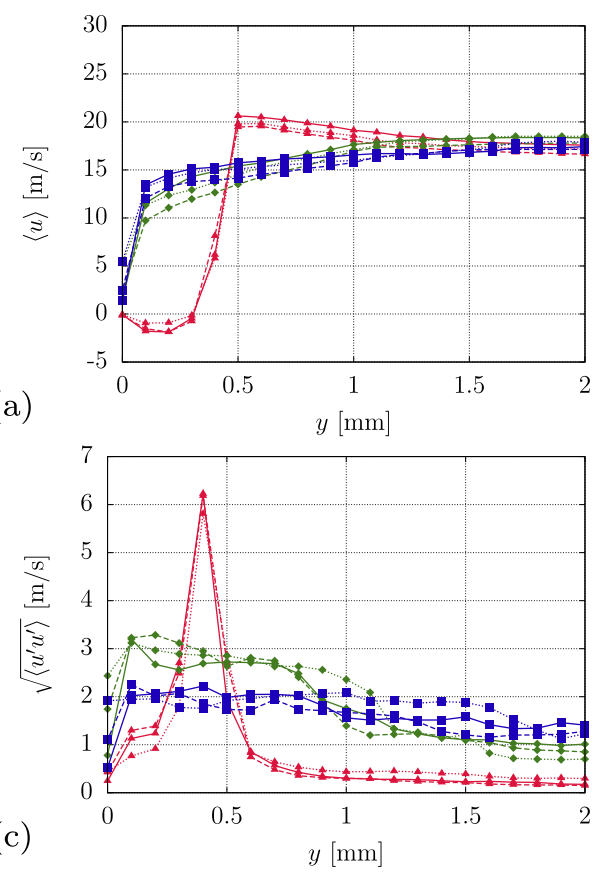

(b)

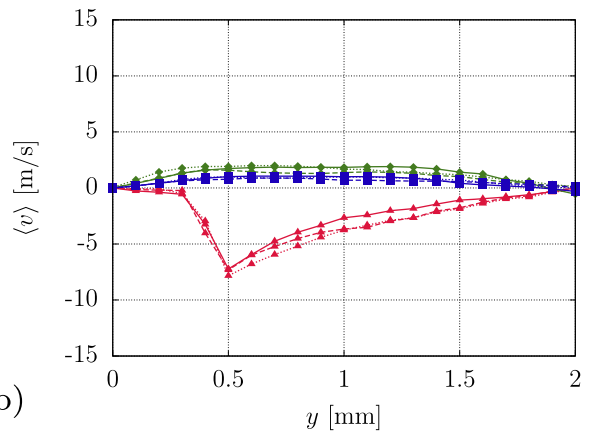

(d)

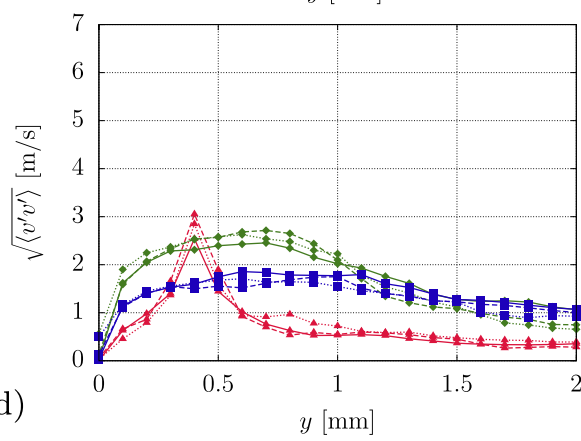

FIG. 22. Grid convergence study: mean and fluctuating velocity components for $\sigma=0.78$ at positions $x=0.5 \mathrm{~mm}$ (red triangle), $x=8.0 \mathrm{~mm}$ (green diamond), and $x=13.0 \mathrm{~mm}$ (blue square) for grid level 3 (dotted), grid level 4 (dashed), and grid level 5 (solid). 
The level 1 grid is too coarse to predict regions of vapor productions but is used to develop the mean flow field. Already on the level 2 grid, a converged flow behavior is found with respect to the global void fraction. Further refinement to level 3 and higher introduces a lower shedding frequency but does not alter the mean value of the vapor volume fraction. Small scale vapor structures finally are already fully resolved on the level 3 grid.

These findings are also confirmed by the turbulence statistics. Figure 22 shows the mean and fluctuating velocity components for the level 3, level 4, and level 5 grids at different channel positions. We find a good agreement between all three levels.

\section{APPENDIX B: DISCUSSION OF THE INLET BOUNDARY CONDITION}

In this appendix, we discuss the lower level of turbulent fluctuations in the numerically predicted incoming flow field compared to the measurements of Sou et al. ${ }^{38,39}$ Figure 23 summarizes the flow conditions at the nozzle inlet at $x=0.5 \mathrm{~mm}$ for all investigated cavitation numbers. The mean streamwise flow velocity shows a very good agreement between simulation and experimental data. In contrast, the core flow, which at this position is not affected by the growing boundary layer at the wall, contains a significantly lower level of velocity fluctuations in the LES. Especially the $v^{\prime}$ fluctuations are considerably larger in the experiment.

To quantify effects of boundary conditions in the inflow section, we conducted a simulation of the $\sigma=0.78$ configuration including a periodic precursor simulation, as sketched in Fig. 24. The length of the precursor domain is $\Delta x=0.01 \mathrm{~m}$. A forcing term is applied on the periodic precursor domain to maintain an average bulk velocity $u_{b, \sigma=0.78}=2.0 \mathrm{~m} / \mathrm{s}$. The instantaneous velocity field is then mapped onto the inlet boundary of the nozzle domain.

Fig. 25 shows the velocity components at the duct inlet for the experiment, for the LES with a prescribed laminar profile, and for the LES with precursor simulation. Very little difference is observed between the two simulation results. The thickness of the shear layer and the level of streamwise velocity fluctuations are essentially unaltered. $v^{\prime}$ fluctuation components are slightly
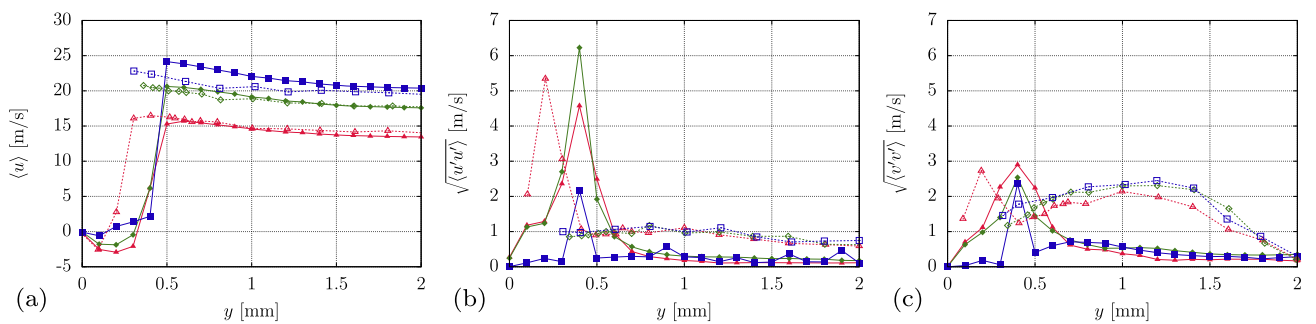

FIG. 23. Mean and fluctuating velocity components at the nozzle inlet $(x=0.5 \mathrm{~mm}$ ) for $\sigma=1.27$ (red triangle), $\sigma=0.78$ (green diamond), and $\sigma=0.65$ (blue square); filled symbols/solid lines represent simulation results, empty symbols/dashed lines are experimental results by Sou et al. . $^{38,39}$

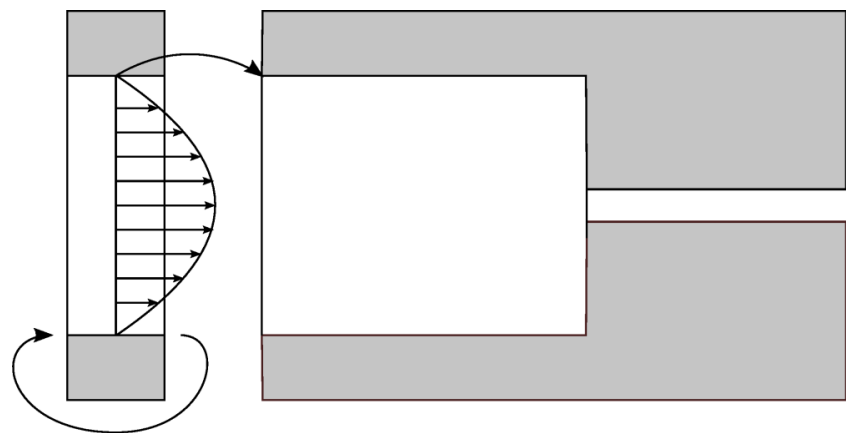

FIG. 24. Schematic of the mapping strategy in the nozzle LES with precursor simulation. 

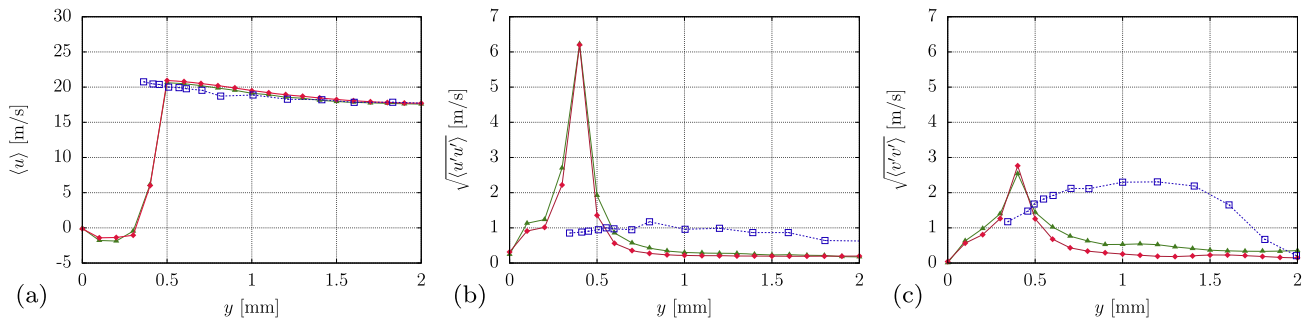

FIG. 25. Mean and fluctuating velocity components at the nozzle inlet $(x=0.5 \mathrm{~mm})$ for $\sigma=0.78$ with laminar inflow profile (green triangle), fully developed inflow from precursor simulation (red diamond), and experiment (blue square); filled symbols/solid lines represent simulation results, empty symbols/dashed lines are experimental results by Sou et al. ${ }^{38,39}$

increased with a precursor simulation but follow the trend of the results obtained without a precursor and with a prescribed laminar profile.

We therefore believe that the high level of turbulence intensity does not originate from grid under-resolution of an otherwise turbulent duct flow. Note that the Reynolds number $R_{\tau}$ based on the friction velocity and the boundary layer thickness of the nozzle flow is on the order of $R e_{\tau} \sim 100$. A more likely cause are disturbances induced by the pumping system, and by flow deflections and throttles and pipes connecting the nozzle domain with the experimental apparatus. These uncertainties cannot be accounted for in the LES without additional evaluation of the entire upstream flow.

${ }^{1}$ C. E. Brennen, Cavitation and Bubble Dynamics (Cambridge University Press, 1995).

${ }^{2} \mathrm{~S}$. Fujikawa and T. Akamatsu, "Effects of the non-equilibrium condensation of vapour on the pressure wave produced by the collapse of a bubble in a liquid," J. Fluid Mech. 97, 481-512 (1980).

${ }^{3}$ Y. Tomita and A. Shima, "Mechanisms of impulsive pressure generation and damage pit formation by bubble collapse," J. Fluid Mech. 169, 535-564 (1986).

${ }^{4}$ A. Vogel, W. Lauterborn, and R. Timm, "Optical and acoustic investigations of the dynamics of laser-produced cavitation bubbles near a solid boundary," J. Fluid Mech. 206, 299-338 (1989).

${ }^{5}$ A. Philipp and W. Lauterborn, "Cavitation erosion by single laser-produced bubbles," J. Fluid Mech. 361, 75-116 (1998).

${ }^{6}$ C.-D. Ohl, M. Arora, R. Dijkink, V. Janve, and D. Lohse, "Surface cleaning from laser-induced cavitation bubbles," Appl. Phys. Lett. 89, 074102-1-074102-3 (2006).

${ }^{7}$ R. D. Reitz, "Mechanism of atomization of a liquid jet," Phys. Fluids 25, 1730 (1982).

${ }^{8}$ S. P. Lin and R. D. Reitz, "Drop and spray formation from a liquid jet," Annu. Rev. Fluid Mech. 30, 85-105 (1998).

${ }^{9}$ N. Tamaki, M. Shimizu, K. Nishida, and H. Hiroyasu, "Effects of cavitation and internal flow on atomization of a liquid jet," Atomization Sprays 8, 179-197 (1998).

${ }^{10}$ N. Tamaki, M. Shimizu, and H. Hiroyasu, "Enhancement of the atomization of a liquid jet by cavitation in a nozzle hole," Atomization Sprays 11, 125-137 (2001).

${ }^{11}$ F. Payri, V. Bermudez, F. Payri, and F. Salvador, "The influence of cavitation on the internal flow and the spray characteristics in diesel injection nozzles," Fuel 83, 419-431 (2004).

12 M. Dular, B. Bachert, B. Stoffel, and B. Širok, "Relationship between cavitation structures and cavitation damage," Wear 257, 1176-1184 (2004).

${ }^{13}$ O. Asi, "Failure of a diesel engine injector nozzle by cavitation damage," Eng. Failure Anal. 13, 1126-1133 (2006).

${ }^{14}$ W. Bergwerk, "Flow pattern in diesel nozzle spray holes," Proc. Inst. Mech. Eng. 173, 655-660 (1959).

15 T. Momma and A. Lichtarowicz, "A study of pressures and erosion produced by collapsing cavitation," Wear 186, 425-436 (1995).

${ }^{16}$ J.-P. Franc, M. Riondet, A. Karimi, and G. L. Chahine, "Material and velocity effects on cavitation erosion pitting," Wear 274-275, 248-259 (2012).

${ }^{17}$ M. S. Plesset and R. B. Chapman, "Collapse of an initially spherical vapour cavity in the neighbourhood of a solid boundary," J. Fluid Mech. 47, 283-290 (1971).

${ }^{18}$ M. S. Plesset and A. Prosperetti, "Bubble dynamics and cavitation,” Annu. Rev. Fluid Mech. 9, 145-185 (1977).

${ }^{19}$ L. d'Agostino and C. E. Brennen, "Linearized dynamics of spherical bubble clouds," J. Fluid Mech. 199, 155-176 (1989).

${ }^{20}$ C. Delale, G. Schnerr, and J. Sauer, "Quasi-one-dimensional steady-state cavitating nozzle flows," J. Fluid Mech. 427, 167-204 (2001).

${ }^{21}$ E. Lauer, X. Y. Hu, S. Hickel, and N. A. Adams, "Numerical investigation of collapsing cavity arrays," Phys. Fluids 24, 052104 (2012).

${ }^{22}$ E. Lauer, X. Y. Hu, S. Hickel, and N. A. Adams, "Numerical modelling and investigation of symmetric and asymmetric cavitation bubble dynamics," Comput. Fluids 69, 1-19 (2012).

${ }^{23}$ E. Giannadakis, M. Gavaises, H. Roth, and C. Arcoumanis, "Cavitation modelling in single-hole diesel injector based on Eulerian-Lagrangian approach," in Proceedings of THIESEL International Conference Thermo- Fluid Dynamic Processes Diesel Engines, Valencia, Spain (La Editorial de la UPV, 2004), pp. 47-60. 
${ }^{24}$ E. Giannadakis, D. Papoulias, M. Gavaises, C. Arcoumanis, C. Soteriou, and W. Tang, "Evaluation of the predictive capability of diesel nozzle cavitation models," SAE Paper 0245, 2007.

${ }^{25}$ C. W. Hirt and B. D. Nichols, "Volume of fluid (VOF) method for the dynamics of free boundaries," J. Comput. Phys. 39 , 201-225 (1981).

${ }^{26}$ R. F. Kunz, D. A. Boger, D. R. Stinebring, T. S. Chyczewski, J. W. Lindau, H. J. Gibeling, S. Venkateswaran, and T. Govindan, "A preconditioned Navier-Stokes method for two-phase flows with application to cavitation prediction," Comput. Fluids 29, 849-875 (2000).

${ }^{27}$ W. Yuan, J. Sauer, and G. H. Schnerr, "Modeling and computation of unsteady cavitation flows in injection nozzles," Mec. Ind. 2, 383-394 (2001).

${ }^{28}$ D. P. Schmidt, "Cavitation in diesel fuel injector nozzles," Ph.D. thesis, University of Wisconsin, Madison, 1997.

${ }^{29}$ D. P. Schmidt, C. J. Rutland, M. L. Corradini, P. Roosen, and O. Genge, "Cavitation in two-dimensional asymmetric nozzles," SAE Technical Paper 1999-01-0518, 1999.

${ }^{30}$ G. H. Schnerr, I. H. Sezal, and S. J. Schmidt, "Numerical investigation of three-dimensional cloud cavitation with special emphasis on collapse induced shock dynamics," Phys. Fluids 20, 040703 (2008).

${ }^{31}$ S. J. Schmidt, M. S. Mihatsch, M. Thalhamer, and N. A. Adams, "Assessment of erosion sensitive areas via compressible simulation of unsteady cavitating flows," in Advanced Experimental and Numerical Techniques for Cavitation Erosion Prediction, edited by K.-H. Kim, G. L. Chahine, J.-P. Franc, and A. Karimi (Springer, 2014), pp. 329-344.

${ }^{32}$ C. P. Egerer, S. Hickel, S. J. Schmidt, and N. A. Adams, "Large-eddy simulation of turbulent cavitating flow in a micro channel," Phys. Fluids 26, 085102 (2014).

${ }^{33}$ A. Andriotis, M. Gavaises, and C. Arcoumanis, "Vortex flow and cavitation in diesel injector nozzles," J. Fluid Mech. 610, 195-215 (2008).

${ }^{34}$ E. Giannadakis, M. Gavaises, and C. Arcoumanis, "Modelling of cavitation in diesel injector nozzles," J. Fluid Mech. 616 153-193 (2008).

${ }^{35}$ D. J. Duke, D. P. Schmidt, K. Neroorkar, A. L. Kastengren, and C. F. Powell, "High-resolution large eddy simulations of cavitating gasoline-ethanol blends," Int. J. Engine Res. 14, 578-589 (2013).

${ }^{36}$ F. J. Salvador, J. Martínez-López, J. V. Romero, and M. D. Roselló, "Computational study of the cavitation phenomenon and its interaction with the turbulence developed in diesel injector nozzles by Large Eddy Simulation (LES)," Math. Comput. Modell. 57, 1656-1662 (2013).

${ }^{37}$ A. Sou, B. Biçer, and A. Tomiyama, "Numerical simulation of incipient cavitation flow in a nozzle of fuel injector," Comput. Fluids 103, 42-48 (2014).

${ }^{38}$ A. Sou, A. Tomiyama, S. Hosokawa, S. Nigorikawa, and T. Maeda, "Cavitation in a two-dimensional nozzle and liquid jet atomization: (LDV measurement of liquid velocity in a nozzle),” JSME Int. J., Ser. B 49, 1253-1259 (2006).

${ }^{39}$ A. Sou, S. Hosokawa, and A. Tomiyama, "Effects of cavitation in a nozzle on liquid jet atomization," Int. J. Heat Mass Transfer 50, 3575-3582 (2007).

${ }^{40}$ S. Som and S. K. Aggarwal, "Effects of primary breakup modeling on spray and combustion characteristics of compression ignition engines," Combust. Flame 157, 1179-1193 (2010).

${ }^{41}$ S. Shibata, S. Nishio, A. Sou, D. Akayama, and M. Mashida, "Evaluation of cavitation in injector nozzle and correlation with liquid atomization," J. Visualization 18, 481-492 (2015).

${ }^{42}$ T. Ménard, S. Tanguy, and A. Berlemont, "Coupling level set/VOF/ghost fluid methods: Validation and application to 3D simulation of the primary break-up of a liquid jet," Int. J. Multiphase Flow 33, 510-524 (2007).

${ }^{43}$ O. Desjardins and H. Pitsch, "Detailed numerical investigation of turbulent atomization of liquid jets," Atomization Sprays 20, 311-336 (2010).

${ }^{44}$ J. K. Dukowicz, "A particle-fluid numerical model for liquid sprays," J. Comput. Phys. 35, 229-253 (1980).

${ }^{45}$ S. Som, A. Ramirez, S. K. Aggarwal, E. M. El-Hannouny, and D. Longman, "Investigation of nozzle flow and cavitation characteristics in a diesel injector," J. Eng. Gas Turbines Power 132, 042802 (2010).

${ }^{46}$ G. Blokkeel, B. Barbeau, and R. Borghi, "A 3D Eulerian model to improve the primary breakup of atomizing jet," SAE Paper No. 2003-01-0005, 2003.

${ }^{47}$ Y. Wang, L. Qiu, R. D. Reitz, and R. Diwakar, "Simulating cavitating liquid jets using a compressible and equilibrium two-phase flow solver," Int. J. Multiphase Flow 63, 52-67 (2014).

${ }^{48}$ R. Saurel, F. Petitpas, and R. Abgrall, "Modelling phase transition in metastable liquids: Application to cavitating and flashing flows," J. Fluid Mech. 607, 313-350 (2008).

${ }^{49}$ R. Saurel, F. Petitpas, and R. A. Berry, "Simple and efficient relaxation methods for interfaces separating compressible fluids, cavitating flows and shocks in multiphase mixtures," J. Comput. Phys. 228, 1678-1712 (2009).

${ }^{50}$ M. Battistoni, S. Som, and D. E. Longman, "Comparison of mixture and multifluid models for in-nozzle cavitation prediction,” J. Eng. Gas Turbines Power 136, 061506 (2014).

${ }^{51}$ J. Ishimoto, F. Sato, and G. Sato, "Computational prediction of the effect of microcavitation on an atomization mechanism in a gasoline injector nozzle," J. Eng. Gas Turbines Power 132, 082801 (2010).

${ }^{52}$ S. Hickel, M. Mihatsch, and S. J. Schmidt, "Implicit large eddy simulation of cavitation in micro channel flows," in Proceedings of the WIMRC 3rd International Cavitation Forum, edited by S. C. Li (University of Warwick, UK, 2011), ISBN: 978-0-9570404-1-0; e-print arXiv:1401.6548.

${ }^{53}$ F. Örley, V. Pasquariello, S. Hickel, and N. A. Adams, "Cut-element based immersed boundary method for moving geometries in compressible liquid flows with cavitation," J. Comput. Phys. 283, 1-22 (2015).

${ }^{54}$ R. Saurel, P. Cocchi, and P. B. Butler, "Numerical study of cavitation in the wake of a hypervelocity underwater projectile," J. Propul. Power 15, 513-522 (1999).

${ }^{55}$ E. Lemmon, M. McLinden, and D. Friend, "Thermophysical properties of fluid systems," in NIST Chemistry WebBook, NIST Standard Reference Database Number 69, edited by P. Linstrom and W. Mallard (National Institute of Standards and Technology, Gaithersburg, MD 20899, 2005), http://webbook.nist.gov (retrieved March 20, 2015).

56 J.-P. Franc and J.-M. Michel, Fundamentals of Cavitation (Springer Science \& Business Media, 2006), Vol. 76. 
${ }^{57}$ R. E. Bensow and G. Bark, "Implicit LES predictions of the cavitating flow on a propeller," J. Fluids Eng. 132, 041302 (2010).

${ }^{58}$ N. A. Adams, S. Hickel, and S. Franz, "Implicit subgrid-scale modeling by adaptive deconvolution,” J. Comput. Phys. 200, 412-431 (2004).

${ }^{59}$ S. Hickel, N. A. Adams, and J. A. Domaradzki, “An adaptive local deconvolution method for implicit LES," J. Comput. Phys. 213, 413-436 (2006).

${ }^{60}$ S. Hickel, C. P. Egerer, and J. Larsson, "Subgrid-scale modeling for implicit large eddy simulation of compressible flows and shock-turbulence interaction," Phys. Fluids 26, 106101 (2014).

${ }^{61}$ S. Hickel, N. A. Adams, and N. N. Mansour, "Implicit subgrid-scale modeling for large-eddy simulation of passive-scalar mixing," Phys. Fluids 19, 095102 (2007).

${ }^{62}$ S. Hickel and N. A. Adams, "On implicit subgrid-scale modeling in wall-bounded flows," Phys. Fluids 19, 105106 (2007)

${ }^{63}$ G. Van Albada, B. Van Leer, and W. Roberts, Jr., "A comparative study of computational methods in cosmic gas dynamics," Astron. Astrophys. 108, 76-84 (1982); abstract available at http://adsabs.harvard.edu/abs/1982A\&amp;A...108...76V.

${ }^{64}$ S. Gottlieb and C.-W. Shu, "Total variation diminishing Runge-Kutta schemes," Math. Comput. Am. Math. Soc. 67(221), 73-85 (1998).

${ }^{65}$ C. P. Egerer, S. Hickel, S. Schmidt, and N. A. Adams, "LES of temporally evolving turbulent cavitating shear layers," in High Performance Computing in Science and Engineering '14 (Springer, 2015), pp. 367-378.

${ }^{66}$ J. Jeong and F. Hussain, “On the identification of a vortex," J. Fluid Mech. 285, 69-94 (1995).

${ }^{67}$ N. Dittakavi and A. Chunekar, "Large eddy simulation of turbulent-cavitation interactions in a Venturi nozzle," J. Fluids Eng. 132, 121301 (2010).

${ }^{68}$ J. Lasheras and E. Hopfinger, "Liquid jet instability and atomization in a coaxial gas stream," Annu. Rev. Fluid Mech. 32 , 275-308 (2000).

${ }^{69}$ E. Villermaux, "Fragmentation,” Annu. Rev. Fluid Mech. 39, 419-446 (2007).

${ }^{70}$ P. Marmottant and E. Villermaux, “On spray formation,” J. Fluid Mech. 498, 73-111 (1999).

${ }^{71}$ S. Dabiri, W. A. Sirignano, and D. D. Joseph, "Interaction between a cavitation bubble and shear flow,” J. Fluid Mech. 651 , $93(2010)$.

72 N. A. Adams and S. J. Schmidt, "Shocks in cavitating flows," in Bubble Dynamics and Shock Waves, edited by C. F. Delale (Springer, 2013), pp. 235-256.

${ }^{73}$ J. Larsson, I. Bermejo-Moreno, and S. K. Lele, "Reynolds- and Mach-number effects in canonical shock-turbulence interaction," J. Fluid Mech. 717, 293-321 (2013).

${ }^{74}$ M. S. Mihatsch, S. J. Schmidt, M. Thalhamer, and N. A. Adams, "Numerical prediction of erosive collapse events in unsteady compressible cavitating flows," in Computational Methods in Applied Sciences, edited by L. Eça, E. Oñate, J. García-Espinosa, T. Kvamsdal, and P. Bergan (Springer, Netherlands, 2013), pp. 187-198.

${ }^{75}$ P. B. Robinson, J. R. Blake, T. Kodama, A. Shima, and Y. Tomita, "Interaction of cavitation bubbles with a free surface," J. Appl. Phys. 89, 8225 (2001).

${ }^{76}$ D. Obreschkow, P. Kobel, N. Dorsaz, A. de Bosset, C. Nicollier, and M. Farhat, "Cavitation bubble dynamics inside liquid drops in microgravity," Phys. Rev. Lett. 97, 204501 (2006).

${ }^{77}$ S. Hickel and J. Larsson, "On implicit turbulence modeling for LES of compressible flows," in Advances in Turbulence XII, edited by B. Eckhardt (Springer, Berlin, 2009), Vol. 132, pp. 873-875. 